\title{
ARTICLE Pharmacokinetics-based identification of pseudoaldosterogenic compounds originating from Glycyrrhiza uralensis roots (Gancao) after dosing LianhuaQingwen capsule
}

Xiao-fang Lan ${ }^{1,2}$, Olajide E. Olaleye ${ }^{2}$, Jun-lan $\mathrm{Lu}^{1,2}$, Wei Yang ${ }^{3}$, Fei-fei Du², Jun-ling Yang ${ }^{2}$, Chen Cheng ${ }^{2}$, Yan-hong Shi ${ }^{2}$,

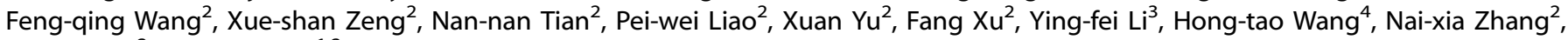
Wei-wei $\mathrm{Jia}^{2}$ and Chuan $\mathrm{Li}^{1,2}$

LianhuaQingwen capsule, prepared from an herbal combination, is officially recommended as treatment for COVID-19 in China. Of the serial pharmacokinetic investigations we designed to facilitate identifying LianhuaQingwen compounds that are likely to be therapeutically important, the current investigation focused on the component Glycyrrhiza uralensis roots (Gancao). Besides its function in COVID-19 treatment, Gancao is able to induce pseudoaldosteronism by inhibiting renal $11 \beta-H S D 2$. Systemic and colonluminal exposure to Gancao compounds were characterized in volunteers receiving LianhuaQingwen and by in vitro metabolism studies. Access of Gancao compounds to $11 \beta$-HSD2 was characterized using human/rat, in vitro transport, and plasma protein binding studies, while $11 \beta$-HSD2 inhibition was assessed using human kidney microsomes. LianhuaQingwen contained a total of 41

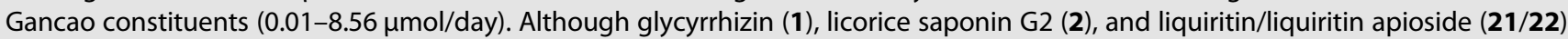
were the major Gancao constituents in LianhuaQingwen, their poor intestinal absorption and access to colonic microbiota resulted in significant levels of their respective deglycosylated metabolites glycyrrhetic acid (8), 24-hydroxyglycyrrhetic acid (M2 $\mathbf{D}$; a new Gancao metabolite), and liquiritigenin (27) in human plasma and feces after dosing. These circulating metabolites were glucuronized/sulfated in the liver and then excreted into bile. Hepatic oxidation of $\mathbf{8}$ also yielded $\mathbf{M} \mathbf{2}_{\mathbf{D}}$. Circulating $\mathbf{8}_{\mathbf{B}}$ and $\mathbf{M} \mathbf{2}_{\mathbf{D}}$, having good membrane permeability, could access (via passive tubular reabsorption) and inhibit renal $11 \beta-H S D 2$. Collectively, $\mathbf{1}$ and $\mathbf{2}$ were metabolically activated to the pseudoaldosterogenic compounds $\mathbf{8}$ and $\mathbf{M} \mathbf{2}_{\mathbf{D}}$. This investigation, together with such investigations of other components, has implications for precisely defining therapeutic benefit of LianhuaQingwen and conditions for its safe use.

Keywords: LianhuaQingwen; glycyrrhetic acid; 24-hydroxyglycyrrhetic acid; Gancao-induced pseudoaldosteronism; licoriceinduced pseudoaldosteronism; $11 \beta$-hydroxysteroid dehydrogenase 2; COVID-19; colonic microbiota

Acta Pharmacologica Sinica (2021) 42:2155-2172; https://doi.org/10.1038/s41401-021-00651-2

\section{INTRODUCTION}

Chinese traditional medicine has played an important role in management of the coronavirus disease 2019 (COVID-19) [1]. LianhuaQingwen capsule is a Chinese herbal medicine recommended in the official guideline for the diagnosis and treatment of COVID-19 [2]. The capsule is prepared from a combination, comprising Forsythia suspensa fruits (Lianqiao), Lonicera japonica flower buds (Jinyinhua), Ephedra sinica stems stir-fried with honey (Zhi-Mahuang), stir-fried Prunus sibirica seeds (Chao-Kuxinren), Gypsum Fibrosum (Shigao), Isatis indigotica roots (Banlangen), Dryopteris crassirhizoma rhizome (Mianmaguanzhong), Houttuynia cordata whole plants (Yuxingcao), Pogostemon cablin overground portion (Guanghuoxiang), Rheum palmatum rhizomes and roots (Dahuang), Rhodiola crenulata rhizomes and roots (Hongjingtian), I-menthol (Bohenao), and Glycyrrhiza uralensis roots (Gancao). In a recent prospective, multicenter, randomized controlled trial of 284 patients with COVID-19, adding LianhuaQingwen to conventional care further improved the recovery rate of symptoms, shortened the time to symptom recovery, and improved the recovery of chest radiologic abnormalities $(P<0.05$ for all) [3]. Two retrospective observational studies showed that adding the capsule to conventional care further accelerated alleviation of the symptoms fever, cough, and fatigue $(P<0.05)[4,5]$. In a cell-based study, LianhuaQingwen dose-dependently reduced production of proinflammatory cytokines at mRNA level, inhibited the severe acute respiratory syndrome coronavirus 2 (SARS-CoV-2) replication, and induced abnormal particle morphology of virion [6]. In addition, several circulating constituents (including rhein, forsythoside A, forsythoside I, and neochlorogenic acid) of LianhuaQingwen were identified to block the host target cell-surface protein angiotensinconverting enzyme 2 receptor [7], to which the SARS-CoV-2 spike protein binds. Like for COVID-19, LianhuaQingwen was also used,

\footnotetext{
${ }^{1}$ Graduate School, Tianjin University of Traditional Chinese Medicine, Tianjin 301617, China; ${ }^{2}$ State Key Laboratory of Drug Research, Shanghai Institute of Materia Medica, Chinese Academy of Sciences, Shanghai 201203, China; ${ }^{3}$ Institute of Chinese Materia Medica, China Academy of Chinese Medical Sciences, Beijing 100700 , China and ${ }^{4}$ Hebei Yiling Chinese Medicine Research Institute, Shijiazhuang 050035, China
}

Correspondence: Wei-wei Jia (weiweijia@simm.ac.cn) or Chuan Li (chli@simm.ac.cn)

Received: 18 January 2021 Accepted: 12 March 2021

Published online: 30 April 2021 
in China, as treatment for other acute viral respiratory illnesses, including 2003 severe acute respiratory syndrome (SARS), 2009 H1N1 swine influenza, 2013 Middle East respiratory syndrome, and 2013 H7N9 avian influenza [8, 9].

Despite extensive use as treatment for acute viral respiratory illnesses, LianhuaQingwen needs to be subjected to more objective rigorous evaluation procedures to provide scientific evidence for its use. An understanding of chemical composition and pharmacokinetics and disposition of key constituents (bioavailable at loci responsible for the therapeutic and/or adverse effects) is vital for translating the potential benefits of complex LianhuaQingwen in rigorous clinical trials. Over the past decade, we have seen great advances in methodology and associated techniques for pharmacokinetic research on such Chinese herbal medicines [10-21]. The field now provides methods that are ripe for use to facilitate identifying the key constituents responsible for therapeutic effects, adverse effects, or drug-drug interactions of such medicines [22-25]. For a systemically acting drug for acute respiratory viral infection, systemic exposure is a prerequisite for drug molecules' access to the loci responsible for the therapeutic effects (such as antiviral effects). Intestinal microbiota plays a vital role in human metabolism, immunity, and reactions to diseases [26]. Acute respiratory viral infection can induce intestinal microbiome alterations and secondary bacterial pneumonia, and for the treatment of patients with COVID-19 is recommended in China to preserve intestinal balance and to prevent secondary bacterial infections [27-29]. Thus, pharmacokinetic research on LianhuaQingwen entails (i) search for herbal compounds circulating significantly (i.e., systemic exposure) after doing the capsule and evaluation of their pharmacokinetics and disposition (including lung distribution) and (ii) evaluation of access of the capsule's compounds to colonic microbiota (i.e., colon-luminal exposure).

As a part of our serial pharmacokinetic investigations of LianhuaQingwen, the current investigation focused on constituents, originating from the component Gancao (licorice), after dosing the capsule. Glycyrrhizin is a known bioactive constituent of Gancao and was found to be active in inhibiting replication of the SARS-associated virus [30]. Notably, pseudoaldosteronism, characterized by hypokalaemia, hypertension, and peripheral edema, could be an adverse effect of consuming Gancaocontaining preparations, by inhibiting renal $11 \beta$-hydroxysteroid dehydrogenase type 2 (11ß-HSD2) [31]. Several glycyrrhizin metabolites, i.e., glycyrrhetic acid-3-O-glucuronide [32-36], glycyrrhetic acid-3-O-sulfate [37, 38], 22a-hydroxyglycyrrhetic acid-3-O-sulfate [37], and 22a-hydroxyglycyrrhetic acid-3-O-sulfate-30-O-glucuronide [39], have been inconsistently proposed to be responsible for or as biomarkers of Gancao-induced pseudoaldosteronism. Given that Gancao is widely used as a component in Chinese herbal medicines and also in dietary products, more research is needed to clarify the compounds responsible for the Gancao-induced adverse effect; this has implication for precisely defining conditions for safe use of LianhuaQingwen. This pharmacokinetic investigation aimed to identify, with respect to systemic exposure and colon-luminal exposure, bioavailable Gancao compounds that are likely to influence the therapeutic outcomes of LianhuaQingwen, particularly potential pseudoaldosterogenic Gancao compounds. Given that the therapeutic action of LianhuaQingwen is also attributed to other components, identified Gancao compounds were not further evaluated in this investigation with respect to their bioactivities associated with the therapeutic action.

\section{MATERIALS AND METHODS}

A detailed description of materials and experimental procedures is provided in the Supplementary Materials and Methods.

\section{Study design}

This investigation of LianhuaQingwen capsule was designed to facilitate identifying Gancao compounds that are likely to be therapeutically important (i.e., bioavailable at loci responsible for drug response of the capsule), particularly pseudoaldosterogenic Gancao compounds. Bioavailable Gancao compounds were identified in human volunteers receiving LianhuaQingwen at label daily dose, with respect to systemic and colon-luminal exposure to the compounds. Because pseudoaldosteronism is a Gancaospecific adverse effect, pseudoaldosterogenic compounds were identified from circulating Gancao compounds of LianhuaQingwen, based on their access to and inhibition of renal $11 \beta$-HSD2. Systemic exposure to Gancao compounds was investigated, based on a full understanding of Gancao-related chemical composition of the dosed LianhuaQingwen. Unchanged and metabolized Gancao compounds in the human plasma and urine samples were characterized by liquid chromatography/mass spectrometry. Gancao metabolites were characterized by various in vitro metabolism studies as well. Colon-luminal exposure to Gancao compounds was characterized by analyzing the human fecal samples and also by in vitro study of metabolism of unabsorbed Gancao constituents by colonic microbiota. Access of circulating Gancao compounds to renal $11 \beta-H S D 2$ was characterized by evaluating their renal clearance ratio, plasma protein binding, membrane permeability, and influences of renal transporters. Inhibition of $11 \beta-H S D 2$ by the circulating Gancao compounds was assessed using human kidney microsomes. Several rat studies were performed to facilitate a better understanding of factors influencing systemic exposure to Gancao metabolites and the metabolites' pulmonary exposure and access to renal $11 \beta-H S D 2$; associated interspecies similarities and differences between rats and humans were evaluated before using the rat data. Literature mining was performed before the preceding in vivo and in vitro studies to avoid missing any potentially important Gancao-related compounds. Fig. 1 summarizes the study workflow.

\section{Literature mining}

Literature mining, for supporting experimental parts of this investigation, was performed to obtain three types of information: (i) various constituents of Glycyrrhiza species and their presence in LianhuaQingwen, (ii) pharmacokinetics, intestinal absorption, and disposition of Gancao compounds and their interactions with colonic microbiota, and (iii) Gancao-induced pseudoaldosteronism. A detailed method for literature mining is described in the Supplementary Materials and Methods.

\section{LianhuaQingwen and study materials}

LianhuaQingwen capsule was manufactured by Shijiazhuang Yiling Pharmaceutical Co., Ltd (Yiling Pharmaceutical; Shijiazhuang, Hebei Province, China) with a Chinese NMPA drug ratification number of GuoYaoZhunZi-Z20040063. Samples of 29 lots of LianhuaQingwen were obtained from Yiling Pharmaceutical to determine Gancao-related chemical composition and lot-to-lot variability. Methods for the chemical composition analysis and for the lot-to-lot variability evaluation are described in subsection 2.9. Samples of the components, including Gancao (G. uralensis roots), were also obtained from Yiling Pharmaceutical. Pure compounds reported to be present in $G$. species were obtained commercially (Supplementary Table S1). 24-Hydroxyglycyrrhetic acid was prepared via anaerobic deglycosylation of licorice saponin G2 using rat colonic microbiota. Glycyrrhetic acid-30-O-glucuronide was prepared via glucuronidation of glycyrrhetic acid using UDPGA-fortified rat liver microsomes.

Modified Gifu anaerobic medium broth was obtained from HyServe (Uffing, Germany). Pooled human liver microsomes (HLM), pooled human intestinal microsomes (HIM), pooled human liver cytosol (HLC), and pooled human intestinal cytosol (HIC) were obtained from Corning Gentest (Woburn, MA, USA). 


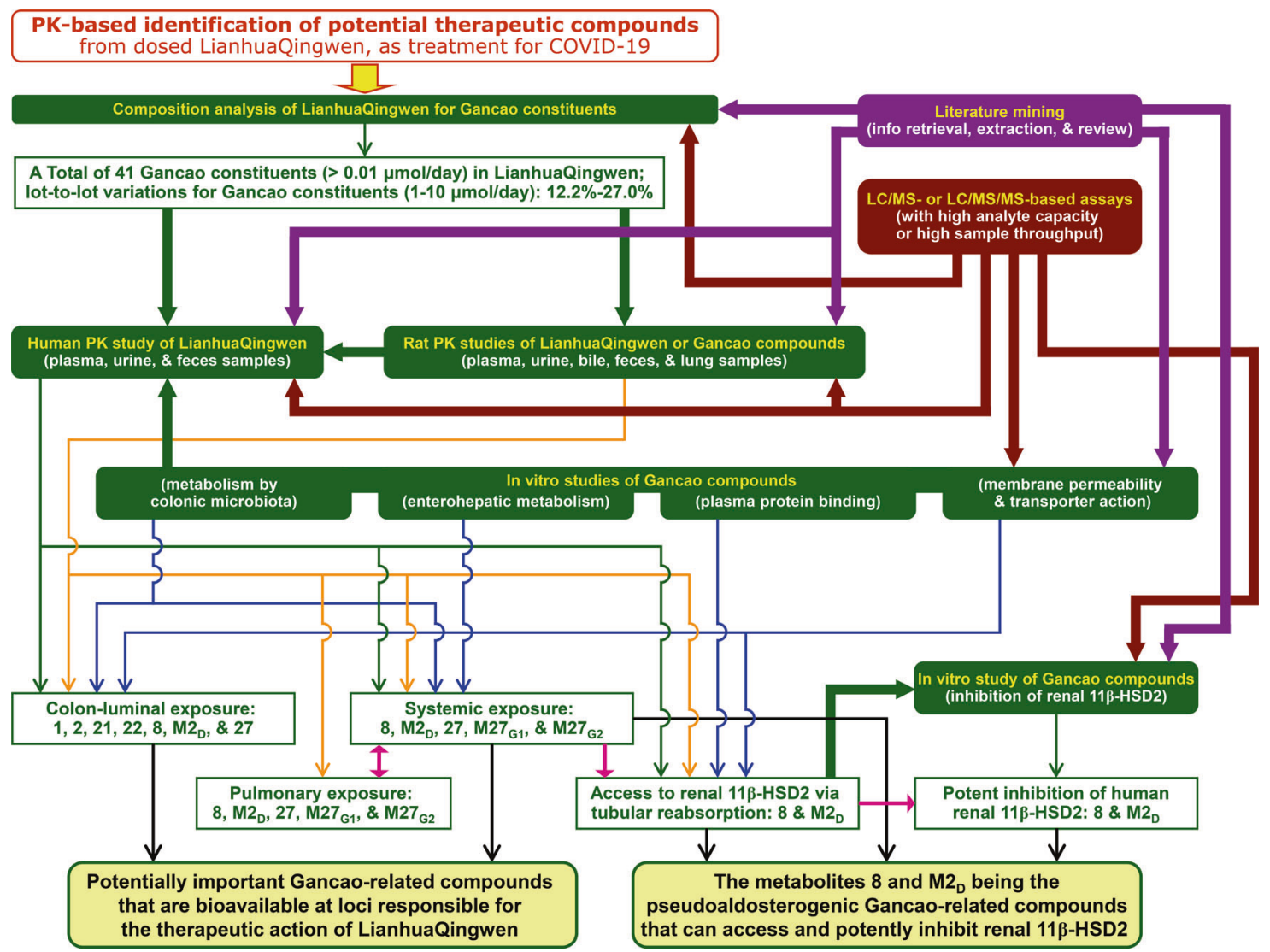

Fig. 1 An overview of approach to pharmacokinetics-based identification of potential therapeutic compounds from LianhuaQingwen, a Chinese herbal used as treatment for COVID-19. The current investigation focused on constituents originating from the component Gancao. PK, pharmacokinetics or pharmacokinetic.

Pooled human kidney microsomes (HKM) was obtained from Celsis In Vitro Technologies (Baltimore, MD, USA) and Sekisui XenoTech (Kansas City, KS, USA); the donor information is shown in Supplementary Table S2. Pooled rat liver microsomes (RLM), pooled rat intestinal microsomes (RIM), pooled rat liver cytosol (RLC), and pooled rat kidney microsomes (RKM) were prepared inhouse from livers, intestines, and kidneys of male Sprague-Dawley rats by differential centrifugation.

HEK-293 cells were obtained from American Type Culture Collection (Manassas, VA, USA). Human solute carrier transporter expression plasmids were constructed commercially. Inside-out membrane vesicles expressing human ATP-binding cassette transporter were purchased from GenoMembrane (Kanazawa, Japan). The positive substrates for these enzymes and transporters were obtained commercially.

Human pharmacokinetic study of LianhuaQingwen

A single-center, open-label pharmacokinetic study of LianhuaQingwen was performed in healthy volunteers at Hebei Yiling Hospital (Shijiazhuang, Hebei Province, China). The protocol for study was reviewed and approved by an ethics committee of clinical investigation at the hospital. The study was registered at the Chinese Clinical Trials Registry (www.chictr.org.cn) with a registration number of ChiCTR1900021460 and performed in accordance with the Declaration of Helsinki. All volunteers gave written informed consent prior to enrollment.

Each participant orally received a single dose of 12 capsules (label daily dose; taking 12 capsules with $240 \mathrm{~mL}$ water within 1 min in three swallows with four capsules each) of LianhuaQingwen on day 1 and days $3-8$. On day 1 , serial blood samples $(5 \mathrm{~mL})$ were collected before and $0.167,0.5,1,3,6,9,12,16,24,28,34$, and $48 \mathrm{~h}$ after dosing the 12 capsules, while serial urine samples were also collected within $0-4,4-10,10-24,24-32$, and 32-48 h periods after dosing. On days 3-7, blood samplings were performed before and $12 \mathrm{~h}$ after dosing the daily 12 capsules. On day 8 , blood and urine samplings were performed according to the respective time schedules on day 1 . On the day just before day 1 (i.e., day 0 ) and on day 9 or 10, fecal samples ( $3-5 \mathrm{~g}$ ) were freshly collected from the participants and immediately mixed with fivefold volumes of the modified Gifu anaerobic medium followed by centrifuging at $1000 \times g$ for $5 \mathrm{~min}$ and the supernatants containing the colonic microbiota were collected under anaerobic conditions to yield individual samples of human colonic microbiota $(\mathrm{HCM})$. The individual samples $(1 \mathrm{~mL})$ on day 0 from the participants were combined to yield a pooled preparation of HCM. All the human study samples were analyzed by liquid chromatography/mass spectrometry.

Supportive in vitro metabolism studies of Gancao compounds The final concentrations of the test substrates in the in vitro metabolism studies were designed to allow the metabolites to be reliably measured. Multiple final concentrations of the test substrates were used for each enzyme kinetic study.

Deglycosylation by colonic microbiota. Glycyrrhizin (1), licorice saponin G2 (2), liquiritin (21), liquiritin apioside (22), isoliquiritin apioside (23), and isoliquiritin (24) were separately dissolved in the modified Gifu anaerobic medium. Before use, the glycosidase activities of pooled HCM and pooled rat colonic microbiota (RCM) preparations were tested under anaerobic conditions using hesperidin as positive substrate [40]. After anaerobic preincubation at $37^{\circ} \mathrm{C}$ for $1 \mathrm{~h}$, the $\mathrm{HCM}$ preparation $(500 \mu \mathrm{L})$ was mixed with 
equal volume of the test compound solution with final compound concentration at $1 \mu \mathrm{mol} / \mathrm{L}$ followed by incubating anaerobically at $37^{\circ} \mathrm{C}$ for $4 \mathrm{~h}$. Sampling was performed at 10 and $30 \mathrm{~min}$ and $4 \mathrm{~h}$ after initiating incubation and the samples were mixed with two volumes of ice-cold methanol. After centrifugation, the supernatant sample was analyzed by liquid chromatography/mass spectrometry. The anaerobic incubation was performed in a Labiophy AL-B anaerobic workstation (Dalian, Liaoning Province, China), inflated with a gas mix of $\sim 15 \%$ carbon dioxide, $83 \%$ nitrogen, and $2 \%$ hydrogen. This study was repeated but by using the pooled preparation of RCM.

Deglycosylation by hepatic glycosidases. Glycyrrhizin (1) at $10 \mu \mathrm{mol} / \mathrm{L}$ was incubated with $\mathrm{HLC}$ at $37^{\circ} \mathrm{C}$ for $20 \mathrm{~h}$ (as described previously [22]) and the resulting incubation sample was analyzed by liquid chromatography/mass spectrometry. This study was repeated but by using RLC.

Oxidation by intestinal and hepatic P450s. Glycyrrhetic acid (8) at $1 \mu \mathrm{mol} / \mathrm{L}$ was incubated with NADPH-fortified HLM at $37^{\circ} \mathrm{C}$ for $1 \mathrm{~h}$ (as described previously [41]) and the resulting incubation sample was analyzed by liquid chromatography/mass spectrometry. This study was repeated but by using NADPH-fortified HIM and RLM. Kinetics of hydroxylation of $\mathbf{8}$ mediated by P450s in HLM and RLM was assessed with respect to Michaelis constant $\left(K_{\mathrm{m}}\right)$, maximum velocity $\left(V_{\max }\right)$, and intrinsic clearance $\left(\mathrm{CL}_{\text {int }}\right)$. The assessment was conducted under linear metabolism conditions by incubation for $15 \mathrm{~min}$, and the test concentrations of 8 were $0.78-100 \mu \mathrm{mol} / \mathrm{L}$ for HLM and RLM.

Conjugation by intestinal and hepatic UGTs and SULTs. Glycyrrhetic acid (8), 24-hydroxyglycyrrhetic acid (M2 $\mathbf{D})$, and liquiritigenin (27), each at $1 \mu \mathrm{mol} / \mathrm{L}$, were separately incubated with UDPGA-fortified HLM at $37^{\circ} \mathrm{C}$ for $1 \mathrm{~h}$ (as described previously [19, 25]) and the resulting incubation samples were analyzed by liquid chromatography/mass spectrometry. This study was repeated but by using UDPGA-fortified HIM, RLM, and RIM. Kinetics of glucuronidation of 8 mediated by UGTs in HLM and RLM was assessed with respect to $K_{\mathrm{m}}, V_{\text {max }}$, and $\mathrm{CL}_{\text {int }}$. The assessment was conducted under linear metabolism conditions by incubation for $5 \mathrm{~min}$, and the test concentrations of 8 were $1.56-200$ and 0.78-100 $\mu \mathrm{mol} / \mathrm{L}$ for HLM and RLM, respectively.

Glycyrrhetic acid (8), 24-hydroxyglycyrrhetic acid (M2 $)$, glycyrrhetic acid-30-O-glucuronide $\left(\mathbf{M 8}_{\mathbf{G}}\right)$, and liquiritigenin (27) at 1 $\mu \mathrm{mol} / \mathrm{L}$ for each were incubated with PAPS-fortified HLC at $37^{\circ} \mathrm{C}$ for $1 \mathrm{~h}$ (as described previously [42]) and the resulting incubation samples were analyzed by liquid chromatography/mass spectrometry. This study was repeated but by using PAPS-fortified HIC and RLC. Kinetics of sulfation of $\mathbf{8}$ mediated by SULTs in HLC and RLC was assessed with respect to $K_{\mathrm{m}}, V_{\max }$ and $\mathrm{CL}_{\text {int }}$. The assessment was conducted under linear metabolism conditions by incubation for $15 \mathrm{~min}$, and the test concentrations of $\mathbf{8}$ were 0.27-200 and 2.47-200 $\mu \mathrm{mol} / \mathrm{L}$ for HLC and RLC, respectively.

Two-step metabolism by human hepatic enzymes. To assess twostep metabolism of 24-hydroxyglycyrrhetic acid (M2D) (i.e., conjugation $\rightarrow$ conjugation), the compound at $10 \mu \mathrm{mol} / \mathrm{L}$ was first incubated with UDPGA-fortified HLM or PAPS-fortified HLC at $37^{\circ} \mathrm{C}$ for $1 \mathrm{~h}$ and then incubated with PAPS-fortified HLC or UDPGA-fortified $\mathrm{HLM}$, respectively, at $37^{\circ} \mathrm{C}$ for $2 \mathrm{~h}$. The final incubation samples were analyzed by liquid chromatography/ mass spectrometry.

Supportive in vitro transport studies of Gancao compounds Cell cultures and cellular uptake by human OAT1, OAT2, OAT3, OAT4, OCT2, OCTN1, URAT1, PEPT1, and PEPT2 were assessed for glycyrrhetic acid (8), 24-hydroxyglycyrrhetic acid (M2 $)$, glycyrrhizin (1), glycyrrhetic acid-3-O-glucuronide (7), glycyrrhetic
acid-30-O-glucuronide $\left(\mathbf{M 8}_{\mathbf{G}}\right)$, liquiritin (21), liquiritin apioside (22), and liquiritigenin (27), each at $50 \mu \mathrm{mol} / \mathrm{L}$, as described previously [43]. In addition, membrane vesicles expressing human MRP3 or MRP4 were used to assess transport of $\mathbf{1}, \mathbf{7}$, and $\mathbf{M} \mathbf{8}_{\mathbf{G}}$, each at $20 \mu \mathrm{mol} / \mathrm{L}$, as described previously [44]. All the incubation samples were analyzed by liquid chromatography/mass spectrometry.

Supportive in vitro plasma-protein-binding studies of Gancao compounds

Unbound fractions in plasma were assessed for glycyrrhetic acid (8), 24-hydroxyglycyrrhetic acid (M2 ), glycyrrhizin (1), glycyrrhetic acid3-O-glucuronide (7), glycyrrhetic acid-30-O-glucuronide $\left(\mathbf{M 8}_{\mathbf{G}}\right)$, and liquiritigenin (27) using an equilibrium dialysis method, as described previously [45]. In addition, human albumin $(600 \mu \mathrm{mol} / \mathrm{L}), a_{1}$-acid glycoprotein $(10 \mu \mathrm{mol} / \mathrm{L})$, and $\gamma$-globulins $(80 \mu \mathrm{mol} / \mathrm{L})$ was assessed, also using the equilibrium dialysis method, to understand which protein(s) were responsible for binding of $\mathbf{8}$ and $\mathbf{M}_{\mathbf{D}}$ in human plasma. All the test concentrations of Gancao compounds were at $50 \mu \mathrm{mol} / \mathrm{L}$, except for 27 at $10 \mu \mathrm{mol} / \mathrm{L}$. Dialysates and associated plasma/protein preparations were sampled for liquid chromatography/mass spectrometry analysis.

\section{Supportive rat pharmacokinetic studies of LianhuaQingwen}

Rat pharmacokinetic studies of LianhuaQingwen were performed at Laboratory Animal Center of Shanghai Institute of Materia Medica (Shanghai, China). The protocols for studies were reviewed and approved by the Institutional Animal Care and Use Committee with the approval IDs 2018-04-LC-28, 2018-11-LC-29, 2019-01-LC30, and 2019-10-LC-31. Care and use of rats were in compliance with the Guidance for Ethical Treatment of Laboratory Animals (the Ministry of Science and Technology of China, 2006; http:// www.most.gov.cn/fggw/zfwj/zfwj2006/200609/t20060930_54389. $\mathrm{htm})$. Male Sprague-Dawley rats $(0.23-0.28 \mathrm{~kg})$ were obtained from Sino-British SIPPR/BK Laboratory Animal (Shanghai, China). Rats received in-house femoral artery cannulation for blood sampling or bile duct cannulation for bile sampling, as described previously [13]. A total of 64 rats were used in the following five rat studies.

In the first study, six rats were randomly assigned into two groups to receive orally a dose of LianhuaQingwen at $3.78 \mathrm{~g} / \mathrm{kg}$ (containing $8 \mu \mathrm{mol} / \mathrm{kg}$ glycyrrhizin) or glycyrrhizin at $8 \mu \mathrm{mol} / \mathrm{kg}$. The LianhuaQingwen dose $3.78 \mathrm{~g} / \mathrm{kg}$ in rats was nine times the dose translated from the human label daily dose of the capsule $(4.2 \mathrm{~g} /$ day), using a body surface area normalization method [46]. Serial blood samples $(150 \mu \mathrm{L})$ were collected before and $0.083,0.25,0.5,1,2,4,6,8,11,15$, 20 , and $24 \mathrm{~h}$ after dosing and were heparinized and centrifuged to yield plasma samples. This rat study was repeated twice, except for the glycyrrhizin administration group.

In the second study, six rats orally received a dose of LianhuaQingwen at $3.78 \mathrm{~g} / \mathrm{kg}$ and serial bile samples were collected within $0-1,1-2,2-4,4-6,6-8,8-10,10-24,24-34$, and $34-48 \mathrm{~h}$ periods after dosing. In addition, four other rats intravenously received a dose of glycyrrhetic acid at $8 \mu \mathrm{mol} / \mathrm{kg}$ and serial bile samples were collected using the preceding time schedule for LianhuaQingwen. During bile collection, an aqueous solution of sodium taurocholate $(7.68 \mathrm{mg} / \mathrm{mL} ; \mathrm{pH} 7.4)$ was infused $(1 \mathrm{~mL} / \mathrm{h})$ into the duodenum.

In the third study, six rats, housed singly in rat metabolic cages (with urine and fecal collection tubes frozen at $-15^{\circ} \mathrm{C}$ ), orally received a dose of LianhuaQingwen at $3.78 \mathrm{~g} / \mathrm{kg}$, and serial urine and fecal samples were collected within $0-8,8-24,24-32$, and $32-48 \mathrm{~h}$ periods after dosing. On the day just before the day initiating the excretion study, feces $(1-2 \mathrm{~g})$ were freshly collected from each rat and immediately mixed with five-fold volumes of the modified Gifu anaerobic medium broth. After centrifuging at $1000 \times g$ for $5 \mathrm{~min}$, the supernatants containing the colonic microbiota were collected under anaerobic conditions to yield 
individual samples of RCM. The resulting individual mixtures were combined to yield a pooled preparation of RCM.

In the fourth study, 30 rats orally received a dose of LianhuaQingwen at $3.78 \mathrm{~g} / \mathrm{kg}$ and killed under isoflurane anesthesia by bleeding from the abdominal aorta at $0,0.25,4,8$, and $11 \mathrm{~h}$ after dosing (six rats per point time) followed by perfusion with $15 \mathrm{~mL}$ saline through the superior vena cava; the blood samples were collected, heparinized, and centrifuged to yield plasma samples. The lungs were excised, rinsed in ice-cold saline, blotted, weighed and homogenized in fivefold (w:w) ice-cold saline.

In the fifth study, six rats, housed singly in rat metabolic cages, intravenously received a dose of glycyrrhetic acid at $8 \mu \mathrm{mol} / \mathrm{kg}$. Serial blood samples were collected before and $0.083,0.25,0.5,1$, $2,4,6,8,11$, and $24 \mathrm{~h}$ after dosing and were heparinized and centrifuged to yield plasma samples, while serial urine samples were also collected within 0-6 and 6-24 h periods.

All the rat study samples were analyzed by liquid chromatography/mass spectrometry.

Composition analysis of LianhuaQingwen for Gancao constituents Composition analysis of LianhuaQingwen for constituents originating from the component Gancao was based on liquid chromatography/mass spectrometry. The analysis was guided by a literature-mined candidate compound list and involved comprehensive detection of constituents present, followed by characterization of the detected constituents and quantification for ranking and grading them. Samples of 29 lots of the capsule were analyzed to evaluate lot-to-lot variability.

Analyses of Gancao compounds and other study compounds in biomatrices

To facilitate pharmacokinetic investigation of LianhuaQingwen for compounds originating from Gancao, two types of liquid chromatography/mass spectrometry-based analysis were performed, i.e., (i) profiling of Gancao compounds (unchanged and metabolized) in human/rat study samples that were prepared with methanol and (ii) quantification of selected Gancao compounds in human/rat study samples that were prepared by acidifying with hydrochloric acid and extracting with ethyl acetate and in in vitro metabolism/transport study samples that were prepared with methanol. Although no internal standard was used, assay validation, implemented according to the European Medicines Agency Guideline on bioanalytical method validation (2012; www. ema.europa.eu), demonstrated that the quantification assays developed were reliable and reproducible for the intended use.

Concentrations of hesperetin, 4-methylumbelliferone, midazolam-1'-hydroxylation, chrysin-7-O-glucuronide, flavone-7-O-sulfate, para-aminohippuric acid, prostaglandin $F_{2 a}$ estrone-3-sulfate, tetraethylammonium, uric acid, glycylsarcosine, estradiol $17 \beta-D-$ glucuronide, and cortisone in in vitro study samples were measured by liquid chromatography/mass spectrometry.

In vitro assessment of $11 \beta-H S D 2$ inhibition by Gancao compounds Several Gancao compounds, including glycyrrhetic acid (8), 24hydroxyglycyrrhetic acid (M2D), glycyrrhizin (1), licorice saponin G2 (2), glycyrrhetic acid-3-O-glucuronide (7), glycyrrhetic acid-30-Oglucuronide $\left(\mathbf{M 8}_{\mathbf{G}}\right)$, and liquiritigenin (27), were assessed for their inhibitory activities on human renal $11 \beta-H S D 2$, the enzymatic activity of which was indicated by biotransformation of cortisol into cortisone. $K_{\mathrm{m}}$ of cortisol for human $11 \beta-\mathrm{HSD} 2$ was measured, using NAD-fortified HKM, to determine the substrate concentration used in inhibition assessment. The inhibition potencies of the test Gancao compounds were initially screened at a concentration of $100 \mu \mathrm{mol} / \mathrm{L}$ and carbenoxolone was used as positive inhibitor [47]. Those Gancao compounds that demonstrated $\geq 50 \%$ inhibition in the initial screening were further evaluated, at multiple concentrations, for their half-maximal inhibitory concentrations $\left(I_{50}\right)$ for $11 \beta-H S D 2$. For the inhibition assessment, NAD-fortified HKM (after 10-min preincubation at $37^{\circ} \mathrm{C}$ ) were incubated with cortisol at $45 \mathrm{nmol} / \mathrm{L}$ (the $K_{\mathrm{m}}$ for human $11 \beta-\mathrm{HSD} 2$ ) for $5 \mathrm{~min}$ (under linear biotransformation condition) in the presence or absence of the test Gancao compound. The biotransformation was terminated by mixing with two volumes of ice-cold methanol. After centrifugation at $21100 \times g$ for $10 \mathrm{~min}$, the supernatant was analyzed for cortisone by liquid chromatography/mass spectrometry. This study was repeated but by using NAD-fortified RKM (containing rat renal $11 \beta-H S D 2)$ with cortisol at $450 \mathrm{nmol} / \mathrm{L}$ (the $K_{\mathrm{m}}$ for the rat enzyme).

\section{Data processing}

After composition analysis, all the detected and characterized Gancao constituents were ranked in descending order according to their respective daily doses (compound doses) and graded into different levels, i.e., $1-10,0.1-1,0.01-0.1$, and $<0.01 \mu \mathrm{mol} /$ day. The compound dose was calculated as the product of the compound's content level in LianhuaQingwen and the capsule's label daily dose $4.2 \mathrm{~g} /$ day.

Pharmacokinetic parameters of Gancao compounds were estimated by noncompartmental analysis using Kinetica (version 5.0; Thermo Scientific, Philadelphia, PA, USA). Renal clearance ratio $\left(R_{\mathrm{rc}}\right)$ was calculated using Eq. 1:

$R_{r c}=C L_{R} /\left(\mathrm{GFR} \times f_{\mathrm{u}-\text { plasma }}\right)$

where $C L_{R}$ is the renal clearance, GFR is the glomerular filtration rate (i.e., 107 and $314 \mathrm{~mL} \cdot \mathrm{h}^{-1} \cdot \mathrm{kg}^{-1}$ for humans and rats, respectively [48]), and $f_{\text {u-plasma }}$ is the unbound fraction in plasma.

GraFit (version 5.0; Erithacus Software, Surrey, UK) was used to estimate the $K_{\mathrm{m}}, V_{\text {max }}$, and $I C_{50}$ values.

Statistical analysis was performed using SPSS Statistics software (version 19.0; IBM, Chicago, IL, USA). A value of $P<0.05$ was considered to be the minimum level of statistical significance.

\section{RESULTS}

Gancao-related chemical composition of LianhuaQingwen and associated lot-to-lot variability of LianhuaQingwen

Literature-mined information supporting this investigation is summarized in the Supplementary Literature-mined information supporting this investigation.

A total of 41 constituents (compound dose, $>0.01 \mu \mathrm{mol} /$ day), originating from the component Gancao, were detected and characterized in samples of LianhuaQingwen, i.e., eight saponins (1-8), 31 flavonoids (21-51), and two coumarins (71 and 72) (Fig. 2 and Supplementary Table S3). These herbal compounds were not detected in any other components of LianhuaQingwen (i.e., 11 herbs, Shigao, or Bohenao). These Gancao constituents in LianhuaQingwen were graded into three levels: $1-10 \mu \mathrm{mol} /$ day [glycyrrhizin (1), licorice saponin G2 (2), licorice saponin H2 (3), liquiritin (21), liquiritin apioside (22), and isoliquiritin apioside (23)], 0.1-1 $\mathrm{mol} /$ day [3 saponins (4-6), 15 flavonoids (24-38)], and 0.01-0.1 $\mu \mathrm{mol} /$ day [the remaining 2 saponins (7 and $\mathbf{8})$, the remaining 13 flavonoids (39-51), and 2 coumarins (71 and 72)]. The ratio of total doses of saponins (1-8), flavonoids (21-51), and coumarins (71 and 72) was 100:98.1:0.5. The doses of 1, 2, and $\mathbf{3}$ (1-10 $\mu \mathrm{mol} / \mathrm{day})$ were $57.4 \%, 18.9 \%$, and $11.9 \%$, respectively, of the total dose of the Gancao saponins in LianhuaQingwen, the remaining saponins 4-6 $(0.1-1 \mu \mathrm{mol} / \mathrm{day})$ and $\mathbf{7 / 8}(0.01-0.1 \mu \mathrm{mol} / \mathrm{day})$ constituted $11.2 \%$ and $0.7 \%$, respectively, of the total dose of the Gancao saponins. The sums of doses of the flavonoids 21-23 (1-10 $\mu \mathrm{mol} /$ day), 24-38 $(0.1-$ $1 \mu \mathrm{mol} / \mathrm{day})$, and 39-51 (0.01-0.1 $\mathrm{mmol} /$ day) were $66.5 \%, 30.0 \%$, and $3.6 \%$, respectively, of the total dose of Gancao flavonoids in LianhuaQingwen. Because only one lot of LianhuaQingwen was used in pharmacokinetic investigation, evaluation of lot-to-lot variability would allow understanding the extent to which the results of pharmacokinetic investigation of the test lot are applicable to other lots of the capsule. LianhuaQingwen exhibited lot-to-lot 


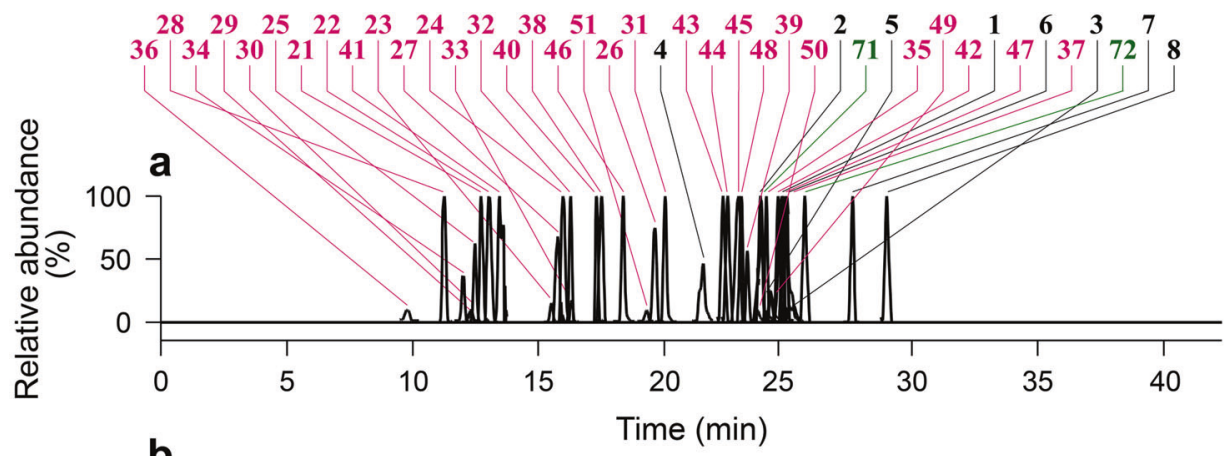

b

Time (min)

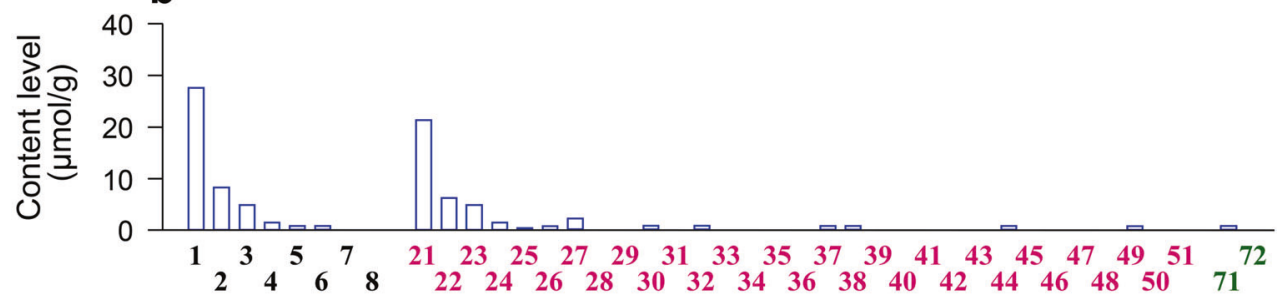

C

Compound ID
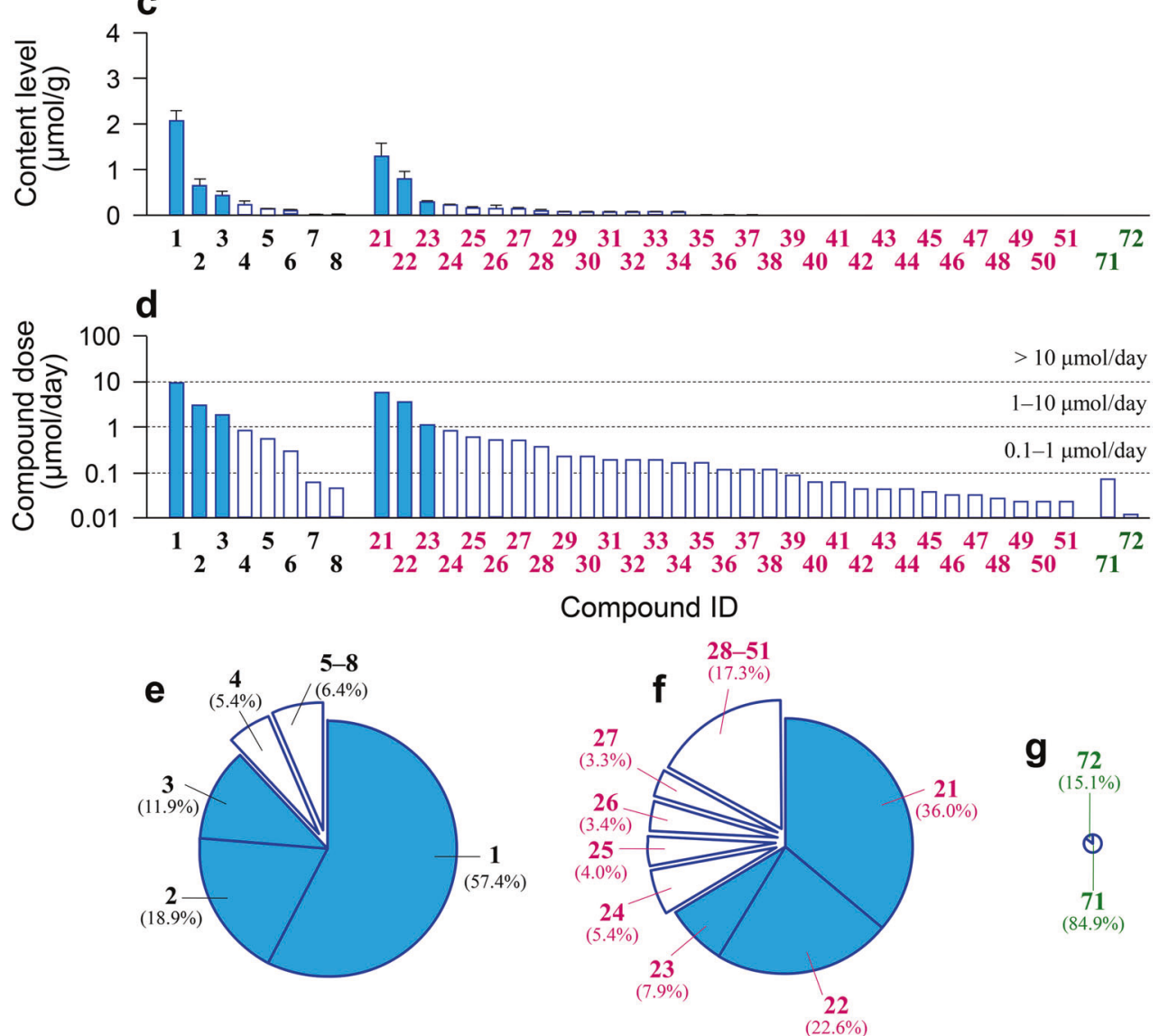

g

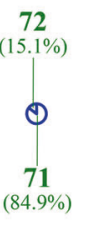

Fig. 2 Gancao constituents detected in LianhuaQingwen. Panel a, stacked liquid chromatograms of Gancao saponins (1-8), flavonoids (21-51), and coumarins (71 and 72), detected by mass spectrometry, in a typical sample of LianhuaQingwen; $\mathbf{b}$, constituents detected in a sample of raw material of Gancao (G. uralensis roots), obtained from Yiling Pharmaceutical Co.; c, mean content levels of Gancao constituents detected in samples of 29 lots of LianhuaQingwen; d, daily doses of the Gancao constituents from the lot A1802055 of LianhuaQingwen at the label daily dose $4.2 \mathrm{~g} /$ day; e, percentage daily doses of saponins in the total daily dose of all the Gancao saponins detected in LianhuaQingwen; $\mathbf{f}$, percentage daily doses of flavonoids in the total daily dose of all the Gancao flavonoids detected in LianhuaQingwen; $\mathbf{g}$, percentage daily doses of coumarins in the total daily dose of all the Gancao coumarins detected in LianhuaQingwen. 1, glycyrrhizin; 2, licorice saponin G2; $\mathbf{3}$, licorice saponin H2; 21, liquiritin; 22, liquiritin apioside; 23, isoliquiritin apioside. The names of 4-8 and 24-51 are shown in Supplementary Table S3. The details of composition analysis of LianhuaQingwen for Gancao constituents are described in the Supplementary Materials and Methods. 
variations of $12.2 \%-27.0 \%, 8.4 \%-38.6 \%$, and $12.2 \%-53.1 \%$ for constituents having 1-10, $0.1-1$, and $0.01-0.1 \mu \mathrm{mol} /$ day doses, respectively (Supplementary Table S3).

Human systemic exposure to Gancao compounds after dosing LianhuaQingwen and their plasma pharmacokinetics and fecal excretion

A total of 14 volunteers (male, m1-m8; female, f9-f14) were recruited for pharmacokinetic study of LianhuaQingwen; among them were three male volunteers with appendectomy (m6-m8) and two female volunteers with constipation ( $f 13$ and $f 14$; i.e., defecation every 2-3 days). During the human study periods, no serious adverse event was observed in volunteers orally receiving LianhuaQingwen. Urine samples from the volunteer $\mathrm{m} 5$ failed to be collected completely, according to the time schedule.

Both unchanged and metabolized Gancao compounds were detected in human plasma samples after dosing LianhuaQingwen (Fig. 3, Table 1, and Supplementary Fig. S1). Glycyrrhetic acid [8; mainly occurring as a metabolite of glycyrrhizin (1)] and 24hydoxyglycyrrhetic acid [M2 $\mathbf{D}$; a metabolite of licorice saponin G2 (2) and 8] were the most abundant of circulating Gancao saponinrelated compounds (sapogenins), while the flavonoid liquiritigenin [27; mainly occurring as a metabolite of liquiritin/liquiritin apioside $(\mathbf{2 1 / 2 2})$ and its metabolites liquiritigenin-7-O-glucuronide $\left(\mathbf{M}_{\mathbf{2}} \mathbf{7}_{\mathbf{G 1}}\right)$ and liquiritigenin-4'-O-glucuronide $\left(\mathbf{M}^{2} \mathbf{7}_{\mathbf{G} 2}\right)$ also circulated significantly in the bloodstream. The other circulating Gancao compounds, i.e., 1, 2, glycyrrhetic acid-30-O-glucuronide ( $\mathbf{M 8}_{\mathbf{G}}$; a metabolite of $\mathbf{8}$ ), 21, 22, isoliquiritin apioside (23), isoliquiritin (24), and isoliquiritigenin (26; mainly occurring as a metabolite of 23/24), were detected at low levels. Gancao constituents with a compound dose $<1 \mu \mathrm{mol} /$ day were negligibly or not detected in the human plasma samples, except $\mathbf{8}, \mathbf{2 6}$, and $\mathbf{2 7}$. These circulating Gancao compounds, except $\mathbf{M} \mathbf{2}_{\mathbf{D}}$, were also detected in the human urine samples. Davidigenin ( $\mathbf{M} 2 \mathbf{7}_{\mathbf{R}}$, a metabolite of $\mathbf{2 7}$ ) was detected only in the urine samples, but its glucuronized or sulfated metabolite was not detected. Notably, compounds reported by others to have inhibitory activities on $11 \beta$-HSD2, i.e., glycyrrhetic acid-3-O-glucuronide (7), glycyrrhetic acid-3-O-sulfate (M8s), 22ahydroxyglycyrrhetic acid-3-O-sulfate, and 22a-hydroxyglycyrrhetic acid-3-O-sulfate-30-O-glucuronide [32-39], were not detected in the plasma or urine samples after dosing LianhuaQingwen.

Table 2 summarizes human pharmacokinetics of glycyrrhetic acid (8) and liquiritigenin (27) in volunteers orally receiving LianhuaQingwen at 12 capsules/day. As shown in Fig. 4(a-d), plasma concentration-time curves of $\mathbf{8}$ were unimodal, with peak concentrations occurring $9-24 \mathrm{~h}\left(T_{\text {peak }}\right)$ and varying lag times of 1-12 $\mathrm{h}$ in detection after dosing LianhuaQingwen (except for the male volunteer $\mathrm{m} 1$ without such a lag time). The lag times resulted from limited dose of the constituent $\mathbf{8}$ from dosed LianhuaQingwen; the peak of $\mathbf{8}$ occurred due to colonic absorption of the microbial metabolite $\mathbf{8}$ via deglycosylation of unabsorbed constituent glycyrrhizin (1) of the dosed capsule. Significant interindividual differences were observed in level of systemic exposure to $\mathbf{8}$. Male volunteers with appendectomy (m6-m8) exhibited lower $A_{U} C_{0-48 h}$ values (corrected for body weight) than the other male volunteers $(\mathrm{m} 1-\mathrm{m} 5)(P<0.05)$, while female volunteers with constipation ( $f 13$ and $f 14$ ) exhibited the greatest $A \cup C_{0-48 \mathrm{~h}}$ values among all the volunteers. After removing the data of $m 6-m 8, f 13$, and $\mathrm{f} 14$ from comparison, there was no statistically significant gender difference ( $m 1-m 5$ versus f9-f12) in $\mathrm{AUC}_{0-48 \mathrm{~h}}$ of $8(P>0.05)$. Following repeated administration of LianhuaQingwen for 7 consecutive days, $A_{U U C} C_{0-48 h}$ values of 8 on day 8 were smaller than the respective values on day $1(P<0.05$; Table 2), i.e., the former being only $16 \%-67 \%$ of the latter, except for f10 being 99\%. Notably, renal excretion of 8 after dosing LianhuaQingwen was very slow $\left(\mathrm{CL}_{\mathrm{R}}, 0.003-0.09 \mathrm{~mL} \cdot \mathrm{h}^{-1} \cdot \mathrm{kg}^{-1}\right)$ and involved tubular reabsorption, as indicated by renal clearance ratios $\left(R_{\mathrm{rc}}\right)$ of $0.2-0.5$. Compared with 8,24 -hydoxyglycyrrhetic acid ( $\mathbf{M 2}_{\mathbf{D}}$ ) exhibited a similar plasma concentration-time profile and a similar profile of interindividual variation in plasma $A U C_{0-48 h}$ (Fig. 4e-h). Plasma $A U C_{0-48 h}$ of $\mathbf{M}_{\mathbf{D}}$ was only about $17 \%$ of that of 8 after dosing the capsule.

Liquiritigenin (27) exhibited bimodal plasma concentrationtime curves, with the first and second peak concentrations occurring 1-3 $\left(T_{\text {peak-1 }}\right)$ and $6-12 \mathrm{~h}\left(T_{\text {peak-2 }}\right)$, respectively, after dosing LianhuaQingwen (Fig. 4i-I). The first peak of $\mathbf{2 7}$ occurred due to intestinal absorption of the constituent $\mathbf{2 7}$ of dosed LianhuaQingwen, while the second peak corresponds to colonic absorption of the microbial metabolite $\mathbf{2 7}$ via deglycosylation of unabsorbed constituents liquiritin/liquiritin apioside (21/22) of the dosed capsule. No lag time was observed in the detection of plasma $\mathbf{2 7}$, which exhibited a significantly shorter mean residence time (MRT) than $8(P<0.05)$. $\mathrm{AUC}_{0-48 \mathrm{~h}}$ values of $\mathbf{2 7}$ for the volunteers poorly correlated with the respective $A U C_{0-48 h}$ values of $8(r=0.07 ; P>0.05)$. Removing appendixes and constipation appeared not to influence $A_{U} C_{0-48 h}$ of 27 (corrected for body weight); associated gender difference (m1-m8 versus f9-f14) was not significant $(P>0.05)$. During the repeated administration of LianhuaQingwen, $\mathrm{AUC}_{0-48 \mathrm{~h}}$ values of $\mathbf{2 7}$ for volunteers on day $\mathbf{8}$ were not significantly different from their respective values on day $1(P>0.05)$. Renal excretion of $27\left(\mathrm{CL}_{\mathrm{R}}, 21-60 \mathrm{~mL} \cdot \mathrm{h}^{-1} \cdot \mathrm{kg}^{-1}\right)$ was substantially faster than that of $\mathbf{8}(P<0.05)$.

As shown in Fig. 3, glycyrrhetic acid (8) and liquiritigenin (27) were significantly detected in fecal samples collected $24-48 \mathrm{~h}$ after dosing LianhuaQingwen. Meanwhile, glycyrrhizin (1), licorice saponin G2 (2), licorice saponin H2 (3), 24-hydroxyglycyrrhetic acid (M2D), and isoliquiritigenin (26) were also detected in the fecal samples, but at low levels.

In vitro metabolism of Gancao compounds

To confirm the Gancao metabolites detected in humans after dosing LianhuaQingwen and to facilitate understanding the associated interspecies similarities and differences between humans and rats, individual Gancao compounds were assessed in various in vitro metabolism studies and the results are shown in Fig. 5 and in Supplementary Figs. S2 and S3. Under anaerobic conditions, glycyrrhizin (1) was deglycosylated into glycyrrhetic acid (8) using HCM. Although glycyrrhetic acid-3-O-glucuronide (7) was also detected in the sample at $10 \mathrm{~min}$ after initiating incubation, 7 was quickly converted into $\mathbf{8}$. Meanwhile, licorice saponin G2 (2) was also deglycosylated, using HCM, into the aglycone 24-hydroxyglycyrrhetic acid (M2D). Under anaerobic conditions, liquiritin apioside (22) and liquiritin (21) were deglycosylated, using HCM, into their flavonone aglycone liquiritigenin (27), which was then reduced into davidigenin $\left(\mathbf{M} 27_{\mathbf{R}}\right)$. Meanwhile, isoliquiritin apioside (23) and isoliquiritin (24) were also deglycosylated into their chalcone aglycone isoliquiritigenin (26). The conversion of 1 into 8 and those of 22/21 into 27 and then into $\mathbf{M}_{\mathbf{2}} \mathbf{F}_{\mathbf{R}}$ were repeatable using RCM under anaerobic conditions.

Deglycosylation of glycyrrhizin (1) into glycyrrhetic acid-3-Oglucuronide (7) took place using $\mathrm{HLC}$, but this conversion was significantly slower than such conversion using HCM (Fig. 5). No glycyrrhetic acid (8) was detected, even when the incubation of 1 with HLC was maintained for 4 and $20 \mathrm{~h}$. Slow deglycosylation of 1 into 7 also took place using RLC.

As shown in Fig. 5 and in Supplementary Figs. S2 and S3, glucuronidation of glycyrrhetic acid (8) by UDPGA-fortified HLM yielded glycyrrhetic acid-30-O-glucuronide $\left(\mathbf{M 8}_{\mathbf{G}}\right)$, rather than glycyrrhetic acid-3-O-glucuronide (7); this biotransformation took place at low level using UDPGA-fortified HIM. Sulfation of $\mathbf{8}$ by PAPS-fortified HLC produced glycyrrhetic acid-3-O-sulfate ( $\left.\mathbf{M} \mathbf{8}_{\mathbf{s}}\right)$, but this sulfated metabolite was not detected using PAPS-fortified HIC. Both sulfation of $\mathbf{M} \mathbf{8}_{\mathbf{G}}$ and glucuronidation of $\mathbf{M} \mathbf{8}_{\mathbf{S}}$ generated glycyrrhetic acid-30-O-glucuronide-3-O-sulfate $\left(\mathbf{M} \mathbf{8}_{\mathbf{G}-\mathbf{s}}\right)$. Oxidation of 8 by NADPH-fortified HLM yielded 24-hydroxyglycyrrhetic acid 

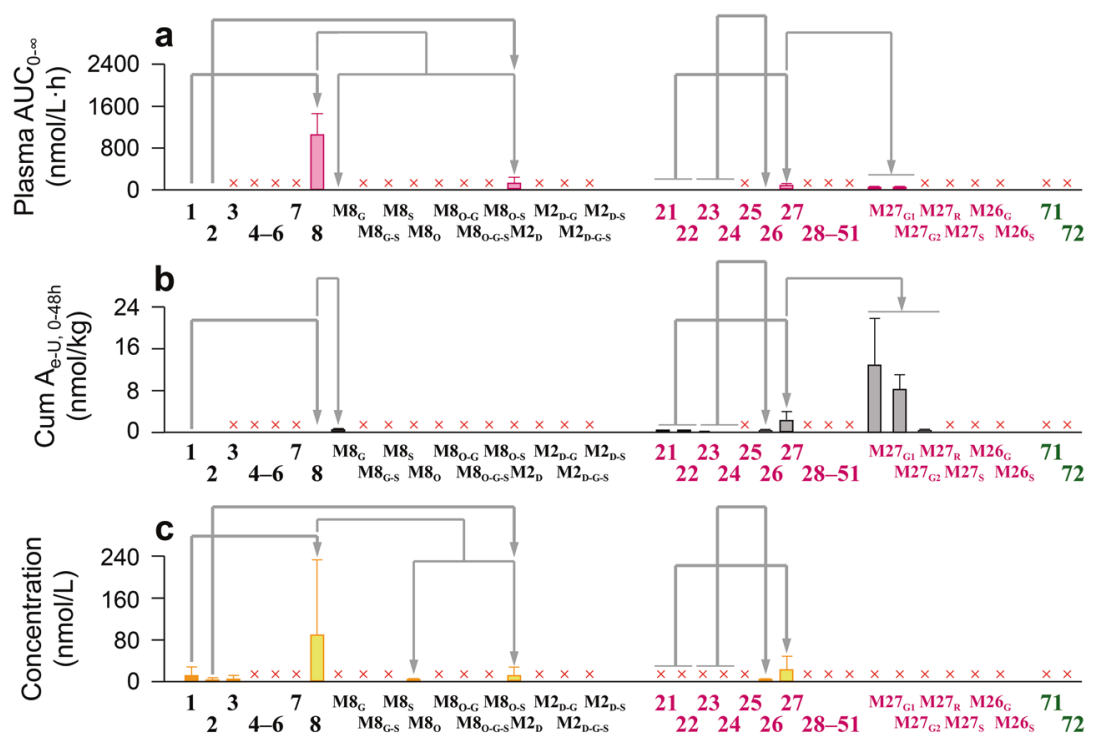

Compound ID
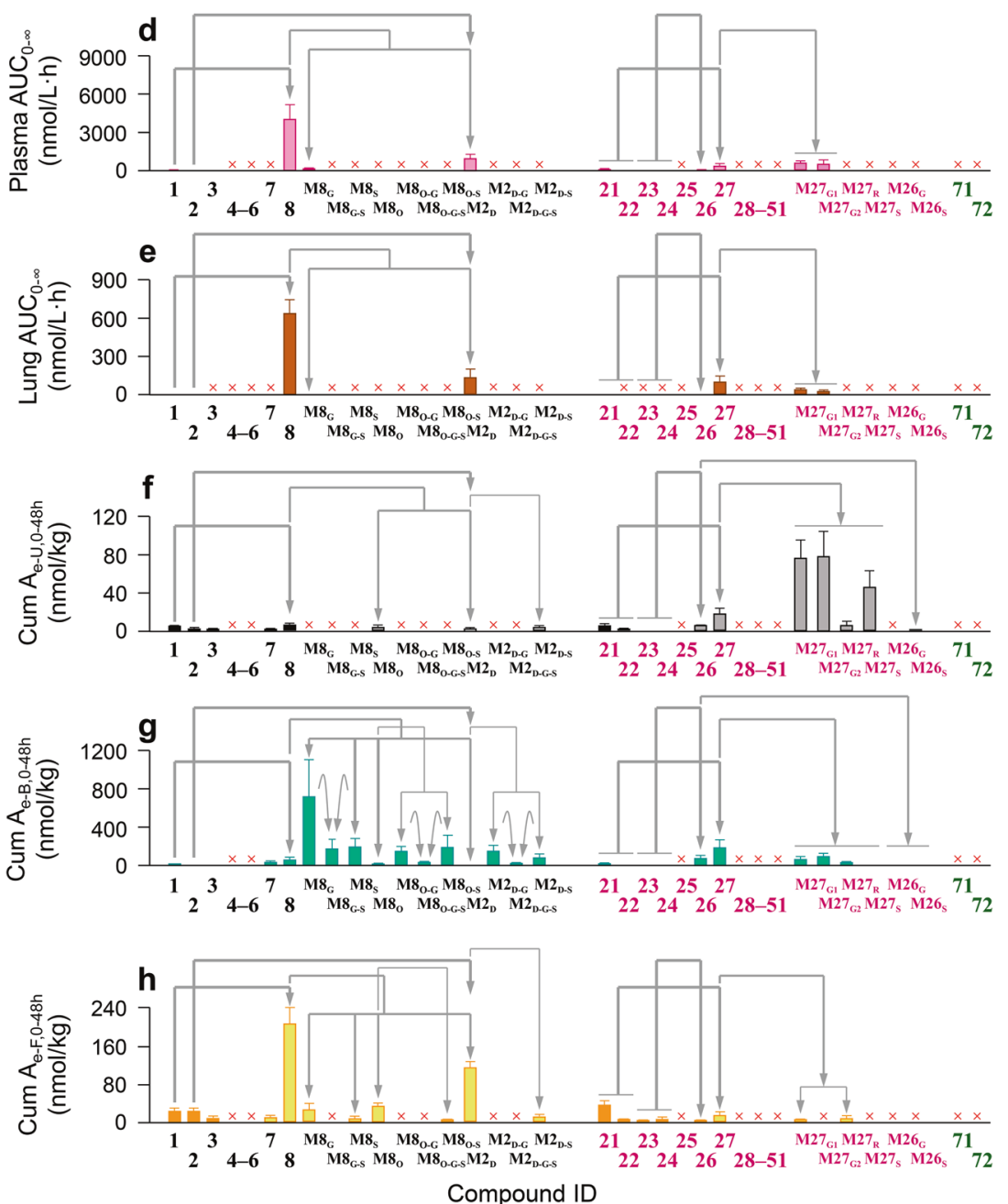

Fig. 3 Gancao compounds detected in human volunteers and rats after orally dosing LianhuaQingwen. Panels a and d, systemic exposure data; $\mathbf{b}$ and $\mathbf{f}$, renal excretion data; $\mathbf{c}$ and $\mathbf{h}$, fecal excretion data; e, lung exposure data; $\mathbf{g}$, hepatobiliary excretion data. The dose for human volunteers was 12 capsules/person (a-c), while that for rats was $3.78 \mathrm{~g} / \mathrm{kg}(\mathbf{d}-\mathbf{h})$. Both unchanged and metabolized Gancao compounds were detected. 1, glycyrrhizin; 7, glycyrrhetic acid-3-O-glucuronide; 8, glycyrrhetic acid; $\mathbf{M 8}_{\mathbf{G}}$, glycyrrhetic acid-30-O-glucuronide; $\mathbf{M} \mathbf{8}_{\mathbf{S}}$, glycyrrhetic acid-3-O-sulfate; $\mathbf{M 2}_{\mathbf{D}}$, 24-hydroxyglycyrrhetic acid; $\mathbf{2 7}$, liquiritigenin; $\mathbf{M 2 7}_{\mathbf{G}}$, liquiritigenin-7-O-glucuronide; $\mathbf{M 2 7}_{\mathbf{G 2}}$, liquiritigenin-4'-Oglucuronide. The names of the other Gancao compounds detected are shown in Table 1. 


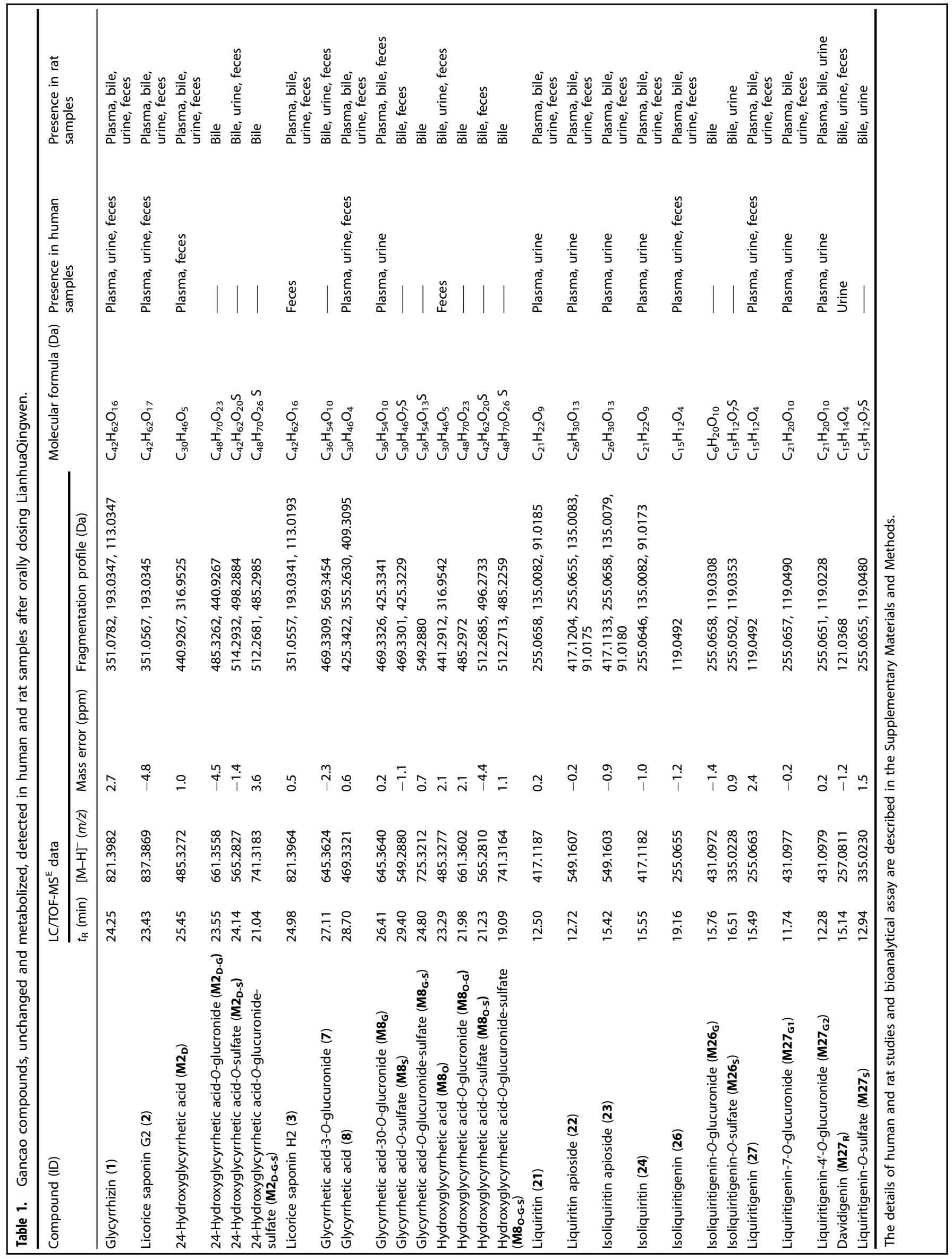


Table 2. Pharmacokinetics of glycyrrhetic acid (8) and liquiritigenin (27) in volunteers orally receiving LianhuaQingwen.

\begin{tabular}{|c|c|c|c|c|}
\hline Pharmacokinetics & Male (m1-m5) & Male (m6-m8) & Female (f9-f12) & Female (f13-f14) \\
\hline \multicolumn{5}{|l|}{ Glycyrrhetic acid (8) on day 1} \\
\hline$C_{\max }(\mathrm{nmol} / \mathrm{L})$ & $88.2 \pm 21.2$ & $48.5 \pm 16.5$ & $51.2 \pm 32.4$ & $236 / 227$ \\
\hline$T_{\text {peak }}(\mathrm{h})$ & $15.4 \pm 5.6$ & $21.3 \pm 4.6$ & $18.0 \pm 4.0$ & $9.0 / 16.0$ \\
\hline Lag time $(\mathrm{h})$ & $3.6 \pm 2.5$ & $10.0 \pm 1.7$ & $4.3 \pm 3.9$ & $1.0 / 3.0$ \\
\hline $\mathrm{AUC}_{0-48 \mathrm{~h}}(\mathrm{~h} \cdot \mathrm{nmol} / \mathrm{L})$ & $1491 \pm 736$ & $623 \pm 175$ & $709 \pm 431$ & 3835 / 2576 \\
\hline $\mathrm{AUC}_{0-\infty}(\mathrm{h} \cdot \mathrm{nmol} / \mathrm{L})$ & $1645 \pm 904$ & $805 \pm 175$ & $766 \pm 420$ & $4516 / 2583$ \\
\hline MRT (h) & $22.7 \pm 7.0$ & $28.2 \pm 5.4$ & $32.8 \pm 20.2$ & $32.4 / 17.2$ \\
\hline$t_{1 / 2}(\mathrm{~h})$ & $7.3 \pm 4.3$ & $7.0 \pm 1.8$ & $5.5 \pm 2.6$ & $14.5 / 1.9$ \\
\hline Cum. $A_{\mathrm{e}-\mathrm{U}, 0-48 \mathrm{~h}}(\mathrm{nmol} / \mathrm{kg})$ & $0.03 \pm 0.02$ & $0.02 \pm 0.01$ & $0.02 \pm 0.02$ & $0.08 / 0.06$ \\
\hline $\mathrm{CL}_{\mathrm{R}}\left(\mathrm{mL} \cdot \mathrm{h}^{-1} \cdot \mathrm{kg}^{-1}\right)$ & $0.02 \pm 0.01$ & $0.02 \pm 0.0$ & $0.02 \pm 0.01$ & $0.02 / 0.02$ \\
\hline \multicolumn{5}{|l|}{ Glycyrrhetic acid (8) on day 8} \\
\hline$C_{\max }(\mathrm{nmol} / \mathrm{L})$ & $41.6 \pm 26.1$ & $17.0 \pm 0.7$ & $41.5 \pm 40.6$ & $102 / 52.1$ \\
\hline$T_{\text {peak }}(\mathrm{h})$ & $8.9 \pm 8.1$ & $16.1 \pm 13.8$ & $10.0 \pm 6.9$ & $12.0 / 24.0$ \\
\hline $\mathrm{AUC}_{0-48 \mathrm{~h}}(\mathrm{~h} \cdot \mathrm{nmol} / \mathrm{L})$ & $575 \pm 642$ & $193 \pm 37$ & $538 \pm 639$ & 1496 / 1103 \\
\hline $\mathrm{AUC}_{0-\infty}(\mathrm{h} \cdot \mathrm{nmol} / \mathrm{L})$ & $741 \pm 888$ & $193 \pm 37$ & $607 \pm 671$ & 1614 / 1289 \\
\hline MRT (h) & $17.6 \pm 9.6$ & $18.9 \pm 0.5$ & $48.1 \pm 31.9$ & 79.7 / 29.8 \\
\hline$t_{1 / 2}(\mathrm{~h})$ & $6.8 \pm 5.6$ & $5.9 \pm 1.9$ & $5.9 \pm 0.8$ & $5.6 / 13.0$ \\
\hline Cum. $A_{\mathrm{e}-\mathrm{U}, 0-48 \mathrm{~h}}(\mathrm{nmol} / \mathrm{kg})$ & $0.02 \pm 0.02$ & $0.01 \pm 0.01$ & $0.03 \pm 0.02$ & $0.1 / 0.02$ \\
\hline $\mathrm{CL}_{\mathrm{R}}\left(\mathrm{mL} \cdot \mathrm{h}^{-1} \cdot \mathrm{kg}^{-1}\right)$ & $0.04 \pm 0.03$ & $0.05 \pm 0.03$ & $0.1 \pm 0.0$ & $0.04 / 0.02$ \\
\hline \multicolumn{5}{|l|}{ Liquiritigenin (27) on day 1} \\
\hline$C_{\max -1}(\mathrm{nmol} / \mathrm{L})$ & $4.2 \pm 2.2$ & - & $3.8 \pm 1.0$ & $5.6 / 2.9$ \\
\hline$C_{\max -2}(\mathrm{nmol} / \mathrm{L})$ & $10.3 \pm 6.4$ & $6.1 \pm 2.7$ & $7.0 \pm 2.5$ & $10.8 / 10.0$ \\
\hline$T_{\text {peak-1 }}(\mathrm{h})$ & $3.4 \pm 3.3$ & $\longrightarrow$ & $1.0 \pm 0.0$ & $1.0 / 3.0$ \\
\hline$T_{\text {peak-2 }}(\mathrm{h})$ & $9.0 \pm 2.4$ & $10.0 \pm 1.7$ & $11.3 \pm 1.5$ & $6.0 / 9.0$ \\
\hline $\mathrm{AUC}_{0-48 \mathrm{~h}}(\mathrm{~h} \cdot \mathrm{nmol} / \mathrm{L})$ & $47.7 \pm 36.2$ & $36.4 \pm 21.5$ & $49.0 \pm 29.7$ & $50.2 / 37.7$ \\
\hline $\mathrm{AUC}_{0-\infty}(\mathrm{h} \cdot \mathrm{nmol} / \mathrm{L})$ & $54.7 \pm 37.3$ & $60.3 \pm 29.5$ & $64.3 \pm 44.2$ & 57.2 / 40.1 \\
\hline MRT (h) & $14.3 \pm 11.9$ & $23.3 \pm 26.3$ & $13.6 \pm 1.5$ & $35.9 / 7.8$ \\
\hline$t_{1 / 2}(\mathrm{~h})$ & $2.3 \pm 1.0$ & $4.5 \pm 2.1$ & $3.9 \pm 2.1$ & $1.6 / 1.1$ \\
\hline Cum. $A_{\mathrm{e}-\mathrm{U}, 0-48 \mathrm{~h}}(\mathrm{nmol} / \mathrm{kg})$ & $2.7 \pm 2.1$ & $2.0 \pm 0.4$ & $2.3 \pm 1.5$ & $1.3 / 1.5$ \\
\hline $\mathrm{CL}_{\mathrm{R}}\left(\mathrm{mL} \cdot \mathrm{h}^{-1} \cdot \mathrm{kg}^{-1}\right)$ & $46.6 \pm 9.3$ & $39.1 \pm 16.6$ & $58.7 \pm 51.8$ & $22.2 / 36.4$ \\
\hline \multicolumn{5}{|l|}{ Liquiritigenin (27) on day 8} \\
\hline$C_{\max -1}(\mathrm{nmol} / \mathrm{L})$ & $2.0 \pm 0.9$ & - & $3.7 \pm 2.1$ & $7.7 /$ \\
\hline$C_{\max -2}(\mathrm{nmol} / \mathrm{L})$ & $5.7 \pm 3.6$ & $3.6 \pm 1.5$ & $6.0 \pm 3.4$ & $4.8 / 2.4$ \\
\hline$T_{\text {peak-1 }}(\mathrm{h})$ & $2.8 \pm 2.4$ & - & $1.0 \pm 0.0$ & $1.0 /-$ \\
\hline$T_{\text {peak-2 }}(\mathrm{h})$ & $9.6 \pm 2.5$ & $11.0 \pm 1.7$ & $6.8 \pm 2.9$ & $9.0 / 9.0$ \\
\hline $\mathrm{AUC}_{0-48 \mathrm{~h}}(\mathrm{~h} \cdot \mathrm{nmol} / \mathrm{L})$ & $36.7 \pm 36.3$ & $21.8 \pm 12.1$ & $48.3 \pm 32.5$ & 25.8 / 17.8 \\
\hline $\mathrm{AUC}_{0-\infty}(\mathrm{h} \cdot \mathrm{nmol} / \mathrm{L})$ & $51.6 \pm 48.3$ & $26.7 \pm 11.1$ & $58.4 \pm 39.0$ & $38.3 / 42.2$ \\
\hline MRT (h) & $20.6 \pm 23.5$ & $11.2 \pm 3.6$ & $8.4 \pm 3.8$ & $18.5 / 7.6$ \\
\hline$t_{1 / 2}(\mathrm{~h})$ & $5.5 \pm 3.0$ & $3.0 \pm 1.2$ & $3.8 \pm 0.5$ & $4.3 / 8.9$ \\
\hline Cum. $A_{\mathrm{e}-\mathrm{U}, 0-48 \mathrm{~h}}(\mathrm{nmol} / \mathrm{kg})$ & $3.8 \pm 4.3$ & $2.5 \pm 0.5$ & $2.6 \pm 1.7$ & $1.1 / 1.4$ \\
\hline $\mathrm{CL}_{\mathrm{R}}\left(\mathrm{mL} \cdot \mathrm{h}^{-1} \cdot \mathrm{kg}^{-1}\right)$ & $63.7 \pm 39.6$ & $101 \pm 25$ & $38.5 \pm 18.3$ & $28.3 / 32.4$ \\
\hline
\end{tabular}

$C_{\max }$ maximum plasma concentration, $T_{\text {peak }}$ the time taken to achieve the peak plasma concentration, $A U C$ area under the plasma concentration-time curve after dosing, MRT mean residence time, $t_{1 / 2}$ terminal half-life, Cum. $A_{\mathrm{e}-\mathrm{u}}$ cumulative amount excreted into urine, $\mathrm{CL}_{\mathrm{R}}$ renal excretory clearance.

(M2 $\mathbf{D})$ and another hydroxyglycyrrhetic acid (M8 $\mathbf{M}_{\mathbf{O}}$; at a significantly lower level than that of $\mathbf{M 2}_{\mathbf{D}}$ ). These oxidized metabolites were not detected when NADPH-fortified HIM was used. Glucuronidation and sulfation of $\mathbf{M} 2_{\mathbf{D}}$ produced 24-hydroxyglycyrrhetic acid-O-glucuronide (M2 $\left.\mathbf{D}_{\mathbf{D}-\mathbf{G}}\right), 24-$ hydroxyglycyrrhetic acid-O-sulfate (M2 $\mathbf{M}_{\mathbf{D}-\mathbf{s}}$ ), and 24-hydroxyglycyrrhetic acid-O-glucuronide-O-sulfate (M2D-G-s). Although the preceding glucuronidation, sulfation, and oxidation of 8 also occurred with RLM and RLC, interspecies differences were observed in enzyme kinetics between the human and rat enzymes
(Table 3 and Supplementary Fig. S4). In brief, rat hepatic UGT exhibited greater metabolic capability for $\mathbf{8}$ than human hepatic UGT, while human hepatic SULT exhibited greater metabolic capability than rat hepatic SULT. Formation of $\mathbf{M 8}_{\mathbf{O}}$ upon incubating 8 with NADPH-fortified human hepatic P450s was significantly poorer than that with rat hepatic P450s.

Glucuronidation of liquiritigenin (27) by UDPGA-fortified HIM yielded liquiritigenin-7-O-glucuronide $\left(\mathbf{M}_{\mathbf{2}} \mathbf{7}_{\mathbf{G 1}}\right)$ and liquiritigenin4'-O-glucuronide $\left(\mathbf{M}^{\prime} \mathbf{7}_{\mathbf{G} 2}\right.$ ), but only $\mathbf{M} \mathbf{2} \mathbf{7}_{\mathbf{G 1}}$ was formed when 

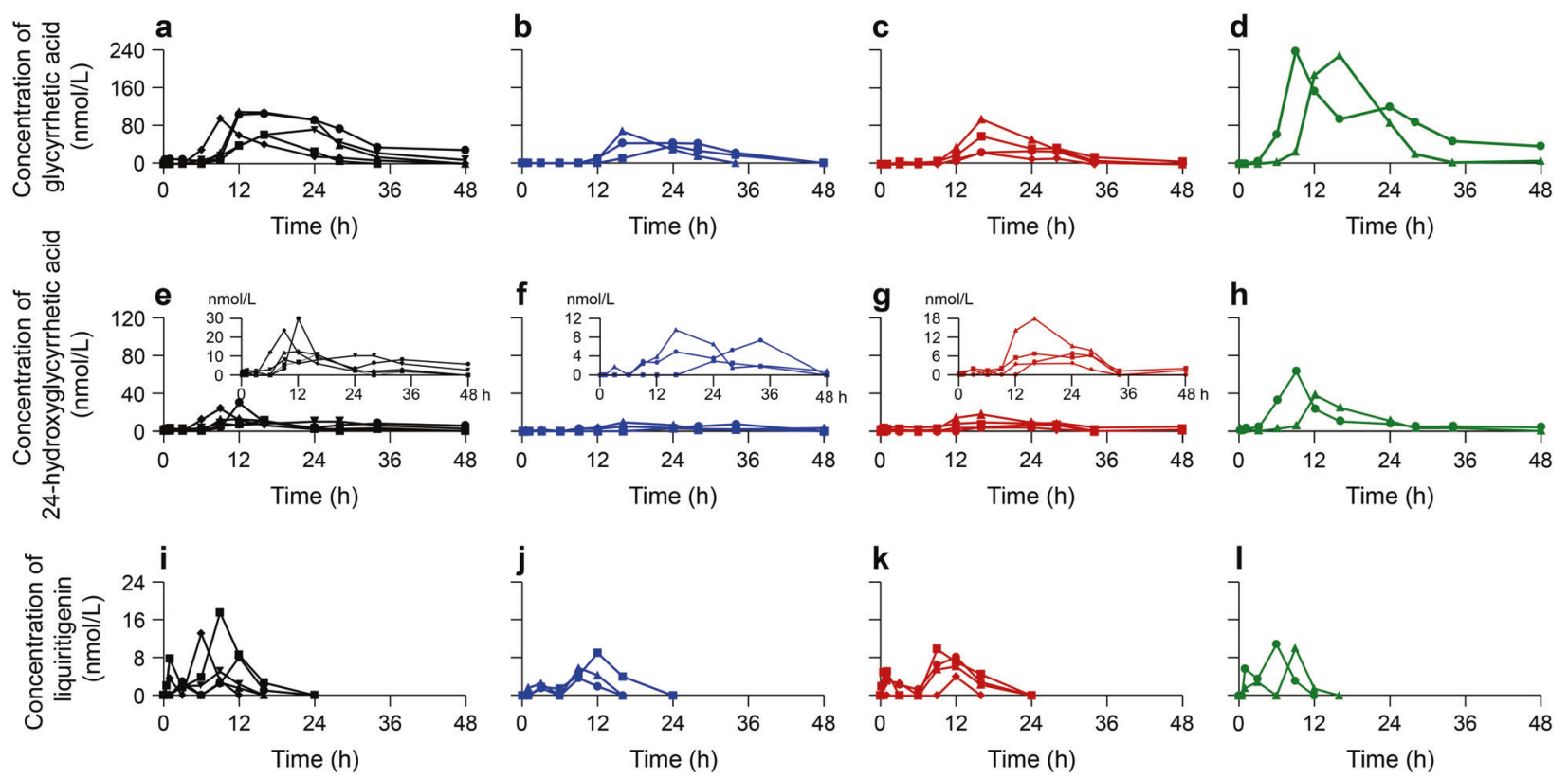

Fig. 4 Plasma concentration-time profiles of Gancao compounds in volunteers (eight males and six females) after dosing LianhuaQingwen at 12 capsules/person. 8, glycyrrhetic acid (a-d); M2 $\mathbf{D}$, 24-hydroxyglycyrrhetic acid (e-h); 27, liquiritigenin (i-l). Black and blue lines, data of males; red and green lines, data of females. Unlike the normal volunteers (black and red lines), three male volunteers (blue lines) had their appendixes removed and two female volunteers (green lines) had infrequent evacuation of the bowels (i.e., defecation every 2-3 days).

UDPGA-fortified HLM was used (Supplementary Fig. S2). Sulfation of $\mathbf{2 7}$ by PAPS-fortified HLC yielded a sulfate of $\mathbf{2 7}(\mathbf{M} \mathbf{2 7}$ s), but at a significantly slower rate than the glucuronidation of $\mathbf{2 7}$. The sulfated metabolite was not detected when PAPS-fortified HIC was used. The preceding glucuronidation and sulfation of $\mathbf{2 7}$ were repeatable using RLM, RLC, and RIM.

Based on the results of human study and those of the in vitro metabolism studies, metabolic pathways of glycyrrhizin (1), liquiritin apioside (22), and liquiritin (21) in humans orally receiving LianhuaQingwen are proposed as shown in Figs. 6 and 7.

Transcellular transport and plasma protein binding of Gancao compounds

To facilitate understanding their renal excretion, individual Gancao compounds were tested in various cellular transport studies and the results are shown in Supplementary Table S4. The circulating Gancao compounds glycyrrhetic acid (8), 24-hydroxyglycyrrhetic acid ( $\left.\mathbf{M} \mathbf{2}_{\mathbf{D}}\right)$, and liquiritigenin (27) exhibited significantly higher rates of membrane permeation in mock cells (transport rate, $\geq 100$ $\mathrm{pmol} \cdot \mathrm{min}^{-1} \cdot \mathrm{mg}^{-1}$ protein) than other test compounds [glycyrrhizin (1), glycyrrhetic acid-3-O-glucuronide (7), glycyrrhetic acid30-O-glucuronide ( $\mathbf{M} \mathbf{8}_{\mathbf{G}}$ ), liquiritin (21), and liquiritin apioside (22); $<5 \mathrm{pmol} \cdot \mathrm{min}^{-1} \cdot \mathrm{mg}^{-1}$ protein]. All these compounds were not substrates of the human renal transporter OAT1, OAT2, OAT3, OCT2, or OCTN1 (responsible for proximal tubular secretion) or OAT4, URAT1, PEPT1, or PEPT2 (responsible for proximal tubular reabsorption), as indicated by the net transport ratios $<3$.

Plasma protein binding is an influencing factor of renal excretion via glomerular filtration. As shown in Table 4, the circulating Gancao compounds glycyrrhetic acid (8) and 24hydroxyglycyrrhetic acid (M2D) were extensively bound to human plasma protein, i.e., albumin (rather than $a_{1}$-acid glycoprotein or $\gamma$ globulins). The compounds related to $\mathbf{8}$, i.e., glycyrrhizin (1), glycyrrhetic acid-3-O-glucuronide (7), and glycyrrhetic acid-30-Oglucuronide $\left(\mathbf{M 8}_{\mathbf{G}}\right.$ ), were also extensively bound to human plasma protein. The circulating Gancao compound liquiritigenin (27) was less bound. Binding profile of rat plasma protein for the preceding compounds was similar to that of human plasma protein (Table 4).
Rat pharmacokinetics of Gancao compounds

Table 1 and Fig. 3 show Gancao compounds (unchanged and metabolized) that were detected in plasma and/or urine samples of rats orally receiving LianhuaQingwen at $3.78 \mathrm{~g} / \mathrm{kg}$. Glycyrrhetic acid (8), 24-hydroxyglycyrrhetic acid (M2 $\mathbf{D}$ ), liquiritigenin (27), liquiritigenin-7-O-glucuronide ( $\left.\mathbf{M} \mathbf{2} \mathbf{7}_{\mathbf{G 1}}\right)$, and liquiritigenin-4'-O-glucuronide $\left(\mathbf{M}_{\mathbf{G}} \mathbf{7}_{\mathbf{G}}\right)$ were major circulating Gancao compounds in rats. These circulating Gancao compounds were also detected in the rat urine samples; urinary $\mathbf{M} \mathbf{2}_{\mathbf{D}}$ was only limitedly detected. Gancao-related compounds reported by others to have inhibitory activities on $11 \beta$ HSD2, including glycyrrhetic acid-3-O-glucuronide (7), glycyrrhetic acid-3-O-sulfate ( $\mathbf{M 8}_{\mathbf{s}}$ ), 22a-hydroxyglycyrrhetic acid-3-O-sulfate, and 22a-hydroxyglycyrrhetic acid-3-O-sulfate-30-O-glucuronide [32-39], were not detected in the rat plasma or urine samples. Based on interspecies similarities between humans and rats in systemic exposure to and renal excretion of Gancao compounds after dosing LianhuaQingwen and associated interspecies similarities in in vitro metabolism and plasma protein binding, several supportive rat studies were performed to obtain additional pharmacokinetic information on the Gancao compounds, particularly 8.

After orally dosing LianhuaQingwen (at $3.78 \mathrm{~g} / \mathrm{kg}$ ) in rats, glycyrrhetic acid (8), 24-hydroxyglycyrrhetic acid (M2D), and liquiritigenin (27) were significantly more abundantly detected in the lungs than other Gancao compounds (Fig. 3). Lung exposure to Gancao compounds after dosing the capsule appeared to be similar to associated systemic exposure.

A total of 28 Gancao compounds (unchanged and metabolized) were detected in bile samples of rats orally receiving a dose of LianhuaQingwen at $3.78 \mathrm{~g} / \mathrm{kg}$ (Fig. 3 and Table 1). The biliary Gancao compounds were absorbed Gancao constituents (1-3 and 21-24), colonic deglycosylated metabolites [mainly glycyrrhetic acid (8), 24-hydroxyglycyrrhetic acid (M2D), and liquiritigenin (27)] of unabsorbed portions of these constituents, and further conjugated metabolites of $\mathbf{8} / \mathbf{M} \mathbf{2}_{\mathrm{D}} / \mathbf{2 7}$. To assess metabolism of $\mathbf{8}$ in vivo, rats received an intravenous bolus dose of pure 8 at $8 \mu \mathrm{mol} /$ $\mathrm{kg}$. Both unchanged $\mathbf{8}$ and its metabolites glycyrrhetic acid-30-Oglucuronide $\left(\mathbf{M 8}_{\mathbf{G}}\right)$, glycyrrhetic acid-3-O-sulfate $\left(\mathbf{M} \mathbf{8}_{\mathbf{s}}\right)$, glycyrrhetic acid-30-O-glucuronide-3-O-sulfate ( $\left.\mathbf{M}_{\mathbf{G}-\mathbf{s}}\right), 24$-hydroxyglycyrrhetic 

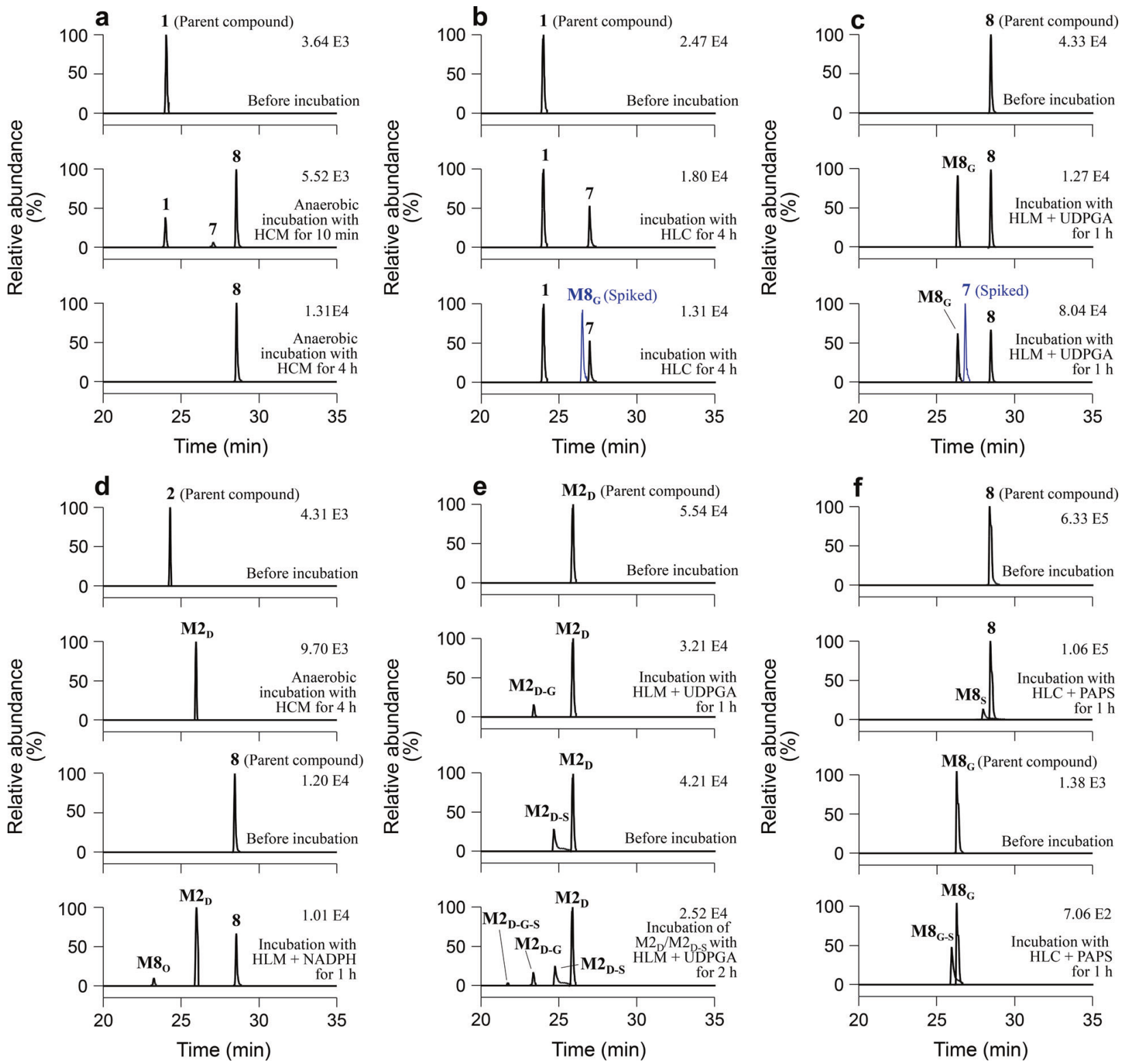

Fig. 5 In vitro metabolism of Gancao compounds, mediated by human enzymes. Panels a and $\mathbf{b}$, deglycosylation of glycyrrhizin (1); c, glucuronidation of glycyrrhetic acid (8); d, deglycosylation of licorice saponin G2 (2)/oxidation of glycyrrhetic acid (8); e, glucuronidation/ sulfation of 24-hydroxyglycyrrhetinic acid (M2 $\mathbf{D})$; $\mathbf{f}$, sulfation of $\mathbf{8} /$ glycyrrhetic acid-30-O-glucuronide (M8 $\mathbf{G})$. 7, glycyrrhetic acid-3-Oglucuronide; $\mathbf{M}_{\mathbf{D}-\mathrm{G},}$ 24-hydroxyglycyrrhetic acid-O-glucuronide; $\mathbf{M 2}_{\mathrm{D}-\mathrm{s},}$ 24-hydroxyglycyrrhetic acid-O-sulfate; $\mathbf{M}_{\mathbf{D}-\mathrm{G}-\mathrm{S}}$, 24-hydroxyglycyrrhetic acid-O-glucuronide- $\mathrm{O}$-sulfate; $\mathbf{M 8}_{\mathbf{G}-\mathbf{s}}$ glycyrrhetic acid-3-O-sulfate-30-O-glucuronide; $\mathbf{M 8}_{\mathbf{S}}$, glycyrrhetic acid-3-O-sulfate; $\mathrm{HCM}$ human colonic microbiota, HLC human liver cytosol, HIM human intestinal microsomes, HLM human liver microsomes, NADPH reduced $\beta$-nicotinamide adenine dinucleotide phosphate, PAPS 3'-phosphoadenosine-5'-phosphosulphate, UDPGA uridine 5'-diphosphoglucuronic acid. Other in vitro metabolism data of Gancao compounds are shown in Supplementary Figs. S2 and S3.

acid [M2 ; also a deglycosylated metabolite of licorice saponin G2 (2)], 24-hydroxyglycyrrhetic acid-O-glucuronide (M2 D-G), 24hydroxyglycyrrhetic acid-O-sulfate (M2 D-s), 24-hydroxyglycyrrhetic acid-O-glucuronide-O-sulfate (M2D-G-s), hydroxyglycyrrhetic acid (M8o), hydroxyglycyrrhetic acid-O-glucuronide (M8 $\mathbf{0 - G}$ ), hydroxyglycyrrhetic acid-O-sulfate (M8 $\mathbf{M}_{\mathbf{o}-\mathbf{s}}$ ), and hydroxyglycyrrhetic acidO-glucuronide-O-sulfate (M8 $\mathbf{0}$-G-s) were detected in bile samples. However, glycyrrhetic acid-3-O-glucuronide (7) was not detected, suggesting it is not a metabolite of $\mathbf{8}$. The biliary metabolites of $\mathbf{8}$ were limitedly or not detected in associated plasma and urine samples. Renal excretion of $\mathbf{8}$ was very slow, with a $\mathrm{CL}_{\mathrm{R}}$ of $0.01 \mathrm{~mL} \cdot \mathrm{h}^{-1} \cdot \mathrm{kg}^{-1}$. Besides glomerular filtration, tubular reabsorption was involved in the renal excretion of $\mathbf{8}$, as indicated by a $R_{\mathrm{rc}}$ of 0.02 .
Unlike synthetic drugs, Chinese herbal medicines are chemically complex. Pharmacokinetics of an herbal compound from an herbal medicine can be affected by the medicine's other coexisting compounds (matrix compounds) via pharmacokinetic drug-drug interactions or drug metabolism; this results in a matrix effect on the pharmacokinetics of the herbal compound. Evaluation of such matrix effects facilitates a more precise understanding of pharmacokinetics of the herbal compound after dosing the medicine. Glycyrrhizin (1) is the most abundant Gancao compound in LianhuaQingwen, while its metabolite glycyrrhetic acid (8) is the most abundant of circulating Gancao compounds after dosing LianhuaQingwen in humans and rats. In this investigation, pharmacokinetic matrix effects of LianhuaQingwen on systemic exposure to $\mathbf{1}$ and glycyrrhetic acid $\mathbf{8}$ were assessed 
in rats by comparing $A \cup C_{0-\infty}$ and $C_{\max }$ after an oral dose of the capsule with the respective data after an oral dose of pure glycyrrhizin at the same dose level. The $A U C_{0-\infty}$ and $C_{\max }$ of 1 after dosing LianhuaQingwen were lower than the respective values after dosing pure glycyrrhizin $(P<0.05)$. However, such pharmacokinetic matrix effects on circulating $\mathbf{8}$ appeared to be limited (Supplementary Fig. S5).

Table 3. $K_{m}, V_{\max }$, and $\mathrm{CL}_{\text {int }}$ data for oxidation, glucuronidation, and sulfation of glycyrrhetic acid (8).

\begin{tabular}{|c|c|c|c|}
\hline Enzyme & $K_{\mathrm{m}}(\mu \mathrm{mol} / \mathrm{L})$ & $\begin{array}{l}V_{\max }\left(\mathrm{pmol} \cdot \mathrm{min}^{-1} \cdot \mathrm{mg}^{-1}\right. \\
\text { protein) }\end{array}$ & $\begin{array}{l}\mathrm{CL}_{\text {int }}\left(\mu \mathrm{L} \cdot \mathrm{min}^{-1} \cdot \mathrm{mg}^{-1}\right. \\
\text { protein) }\end{array}$ \\
\hline \multicolumn{4}{|c|}{ Oxidation of glycyrrhetic acid to yield $\mathbf{M} \mathbf{2}_{D}$ and $\mathbf{M} \mathbf{8}_{O}$} \\
\hline \multirow[t]{2}{*}{ HLM } & $\begin{array}{l}11.3 \pm 1.9 \\
\left(\mathbf{M} \mathbf{2}_{\mathbf{D}}\right)\end{array}$ & $2635.8 \pm 139.4\left(\mathbf{M} \mathbf{2}_{\mathrm{D}}\right)$ & $233.3\left(\mathbf{M} \mathbf{2}_{\mathbf{D}}\right)$ \\
\hline & $\begin{array}{l}67.3 \pm 14.2 \\
\left(\mathbf{M} 8_{0}\right)\end{array}$ & $517.1 \pm 57.8(\mathbf{M 8} \mathbf{o})$ & $7.7\left(\mathbf{M} \mathbf{8}_{\mathbf{0}}\right)$ \\
\hline \multirow[t]{2}{*}{ RLM } & $\begin{array}{l}11.4 \pm 2.1 \\
\left(\mathbf{M} 2_{\mathbf{D}}\right)\end{array}$ & $4793.3 \pm 277.8\left(\mathbf{M} \mathbf{2}_{\mathbf{D}}\right)$ & $420.5\left(\mathbf{M} 2_{D}\right)$ \\
\hline & $\begin{array}{l}33.7 \pm 3.2 \\
\left(\mathbf{M} 8_{\mathbf{o}}\right)\end{array}$ & $2520.4 \pm 100.6(\mathbf{M} 8 \mathbf{0})$ & $74.8\left(\mathbf{M} 8_{0}\right)$ \\
\hline \multicolumn{4}{|c|}{ Glucuronidation of glycyrrhetic acid to yield $\mathbf{M}_{G}$} \\
\hline HLM & $136.0 \pm 31.0$ & $2215.9 \pm 255.4$ & 16.3 \\
\hline RLM & $21.9 \pm 3.3$ & $3221.9 \pm 143.6$ & 147.2 \\
\hline \multicolumn{4}{|c|}{ Sulfation of glycyrrhetic acid to yield $\mathbf{M}_{S}$} \\
\hline HLC & $17.0 \pm 11.5$ & $480.0 \pm 87.8$ & 28.2 \\
\hline RLC & $12.2 \pm 6.2$ & $25.0 \pm 3.5$ & 2.0 \\
\hline
\end{tabular}

Inhibitory potency of Gancao compounds on human $11 \beta-H S D 2$ Using pooled HKM, glycyrrhetic acid (8) and 24hydroxyglycyrrhetic acid (M2D) exhibited significantly potent inhibitory activities on human renal $11 \beta-H S D 2$ compared with the reference compounds glycyrrhizin (1), licorice saponin G2 (2), glycyrrhetic acid-3-O-glucuronide (7), and glycyrrhetic acid-30-Oglucuronide $\left(\mathbf{M 8}_{\mathbf{G}}\right)$, as indicated by the $\mathrm{IC}_{50}$ values (Table 5 ). Gancao flavonoids, including liquiritigenin (27), exhibited poor or negligible inhibition of human $11 \beta$-HSD2 (Supplementary Figs. S6 and S7). Similar differences were observed using pooled RKM (Table 5 and Supplementary Fig. S7). Notably, IC 50 values of the test compounds using pooled HKM from Celsis In Vitro Technologies were significantly lower than respective values using pooled HKM from Sekisui XenoTech (Table 5). There was no $\mathrm{IC}_{50}$ value of $\mathbf{M} \mathbf{2}_{\mathbf{D}}$ for Celsis HKM, because the HKM had run out when the compound was identified in the pharmacokinetic and metabolism studies. Given that only $\mathbf{8}$ and $\mathbf{M} \mathbf{2}_{\mathbf{D}}$ were significantly bioavailable for renal $11 \beta$-HSD2 with potent inhibitory activities on the enzyme, they were considered to be pseudoaldosterogenic Gancao compounds after dosing LianhuaQingwen.

\section{DISCUSSION}

For a complex Chinese herbal medicine, pharmacokinetic research is a useful approach to identifying its potentially important compounds (i.e., compounds bioavailable at loci responsible for the medicine's therapeutic action) and to characterizing their pharmacokinetics and disposition. Based on clinical use of LianhuaQingwen as treatment for the acute viral respiratory illness, such bioavailability of compounds after dosing the capsule was investigated with respect to their systemic and colon-luminal exposure. A total of 41 Gancao constituents (0.01-8.56 $\mu \mathrm{mol} / \mathrm{day})$ were detected and

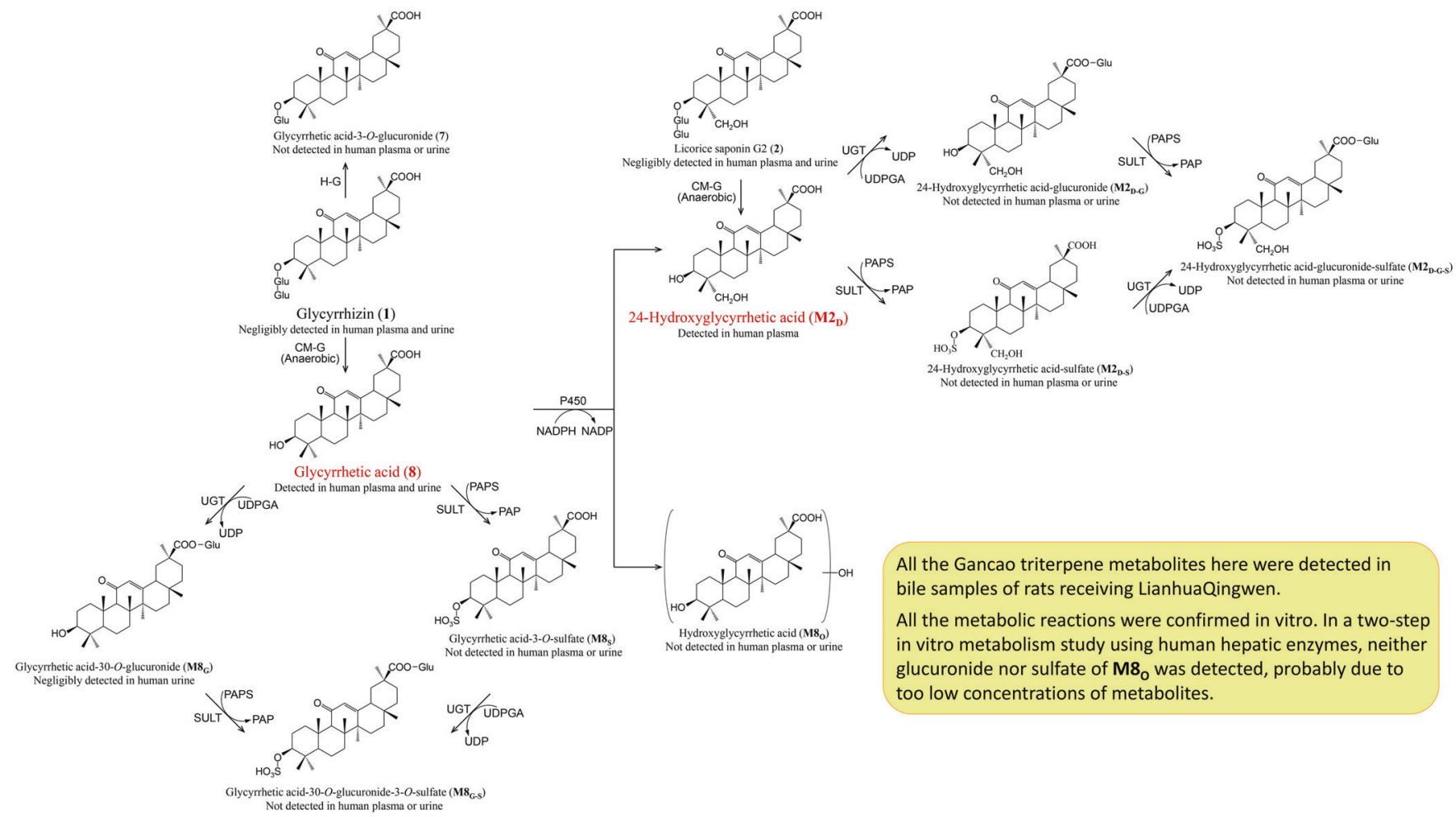

Fig. 6 Proposed metabolic pathways of glycyrrhizin (1) and licorice saponin G2 (2) in humans orally receiving LianhuaQingwen. CM-G glycosidase of colonic microbiota, H-G hepatic glycosidase, P450 cytochrome P450 enzyme, UGT uridine $5^{\prime}$-diphosphoglucuronosyltransferase, SULT sulfotransferase, NADP $\beta$-nicotinamide adenine dinucleotide phosphate, NADPH reduced $\beta$-nicotinamide adenine dinucleotiden phosphate, UDP uridine 5'-diphosphate, UDPGA uridine 5'-diphosphoglucuronic acid, PAP 3'-phosphoadenosine-5'-phosphate, PAPS 3'-phosphoadenosine-5'-phosphosulphate, Glu glucuronosyl. 
<smiles>[14CH3]Oc1ccc(C2CC(=O)C(=O)C3CCC(O)C=C3O2)cc1</smiles>

Liquiritin apioside (22) Detected in human plasma and urine Detected in human plasma and urine Liquiritigenin (27) Detected in human plasma and urine<smiles>COc1ccc2c(c1)OC(c1ccc(O)cc1)CC2=O</smiles>

Liquiritigenin-7-O-glucuronide (M27 Detected in human plasma and urine

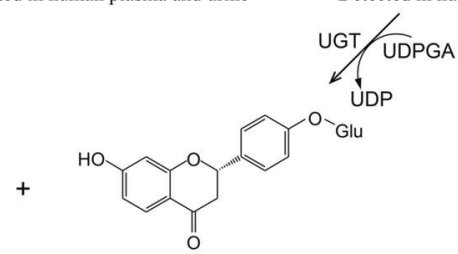
ÙD Detected in human plasma and urine

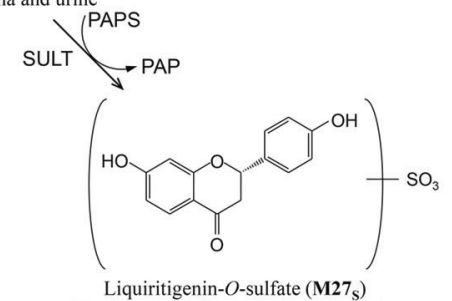

Liquiritigenin- $O$-sulfate (M27 $)$ Not detected in human plasma and urine

\section{All the Gancao flavonone metabolites here were detected in bile samples of rats receiving LianhuaQingwen.}

All the metabolic reactions were confirmed in vitro.

Fig. 7 Proposed metabolic pathways of liquiritin (21) and liquiritin apioside (22) in humans orally receiving LianhuaQingwen. CM-G glycosidase of colonic microbiota, L-G liver glycosidase, UGT uridine 5'-diphosphoglucuronosyltransferase, SULT sulfotransferase, PAP $3^{\prime}$ phosphoadenosine-5'-phosphate, PAPS 3'-phosphoadenosine-5'-phosphosulphate, UDP uridine 5'-diphosphate, UDPGA uridine 5'-diphosphoglucuronic acid, Api apiosyl, Glc glucosyl, Glu glucuronosyl.

Table 4. Unbound fractions of Gancao compounds in human and rat plasma and in human plasma protein solutions $\left(f_{\mathrm{u}}\right)$.

\begin{tabular}{llllll}
\hline Compounds (ID) & \multicolumn{1}{l}{$f_{\mathrm{u}}(\%)$} \\
\cline { 2 - 5 } & Human plasma & Rat plasma & Human albumin & Human $\alpha_{1}$-acid glycoprotein & Human $\gamma$-globulins \\
\hline Glycyrrhetic acid (8) & $0.07 \pm 0.02$ & $0.05 \pm 0.01$ & 0.16 & 100 & 100 \\
24-Hydroxyglycyrrhetic acid (M2 $\mathbf{D})$ & $0.32 \pm 0.02$ & $0.17 \pm 0.01$ & 0.28 & 100 & 100 \\
Glycyrrhizin (1) & $0.6 \pm 0.1$ & $0.4 \pm 0.02$ & - & - & - \\
Glycyrrhetic acid-3-O-glucuronide (7) & $0.07 \pm 0.01$ & $0.09 \pm 0.01$ & - & - & - \\
Glycyrrhetic acid-30-O-glucuronide (M8 & $0.27 \pm 0.02$ & $0.11 \pm 0.01$ & - & - & - \\
Liquiritigenin (27) & $7.7 \pm 2.5$ & $10.7 \pm 3.1$ & - & -
\end{tabular}

Albumin, $\alpha_{1}$-acid glycoprotein, and $\gamma$-globulins in their respective human plasma protein solutions were 600,10 , and $80 \mu$ mol/L, respectively, which minicked their concentrations in normal human plasma.

Table 5. IC $\mathrm{I}_{50}$ for inhibition, by Gancao compounds, of human and rat $11 \beta$-HSD2-mediated dehydrogenation of cortisol.

\begin{tabular}{|c|c|c|c|}
\hline Compounds (ID) & \multicolumn{3}{|l|}{$\mathrm{IC}_{50}(\mu \mathrm{mol} / \mathrm{L})$} \\
\hline Glycyrrhetic acid (8) & $0.0069 \pm 0.0030$ & $0.30 \pm 0.09$ & $0.12 \pm 0.03$ \\
\hline 24-Hydroxyglycyrrhetic acid ( $\left.\mathbf{M} \mathbf{2}_{\mathbf{D}}\right)$ & - & $0.28 \pm 0.06$ & $0.095 \pm 0.047$ \\
\hline Glycyrrhizin (1) & $7.23 \pm 0.94$ & $41.96 \pm 8.69$ & $9.41 \pm 2.84$ \\
\hline Licorice saponin G2 (2) & $14.82 \pm 1.46$ & $24.82 \pm 4.16$ & $61.50 \pm 6.96$ \\
\hline Liquiritigenin (27) & $28.02 \pm 2.23$ & $51.17 \pm 7.45$ & $54.10 \pm 1.05$ \\
\hline
\end{tabular}

The data present mean \pm SD from two independent experiments where each was performed in duplicate (total $n=4$ ), except for those of glycyrrhizin (1), licorice saponin G2 (2), glycyrrhetic acid-30-O-glucuronide ( $\mathbf{M 8}_{\mathbf{G}}$ ), and liquiritigenin (27) for HKM obtained from Celsis In Vitro Technologies with a total $n$ of 2. $\mathrm{IC}_{50}$ value of 24-hydroxyglycyrrhetic acid (M2D) for human 11ß-HSD2 was not measured using HKM from Celsis In Vitro Technologies, because the HKM had run out when the compound was identified as bioavailable for renal 11 $\beta$-HSD2.

characterized in LianhuaQingwen, but only several Gancao metabolites glycyrrhetic acid (8), 24-hydroxyglycyrrhetic acid (M2 ; a new Gancao metabolite), liquiritigenin (27), and liquiritigenin glucuronides $\left(\mathbf{M} \mathbf{2} \mathbf{7}_{\mathbf{G} 1}\right.$ and $\left.\mathbf{M} \mathbf{2} \mathbf{7}_{\mathbf{G} \mathbf{2}}\right)$, rather than their respective parent constituents, exhibited significant systemic exposure after dosing the capsule. These circulating Gancao metabolites also significantly occurred in the lungs of rats receiving LianhuaQingwen. The notable differences in compound count and form between the Gancao-related compounds present in LianhuaQingwen and those in general 


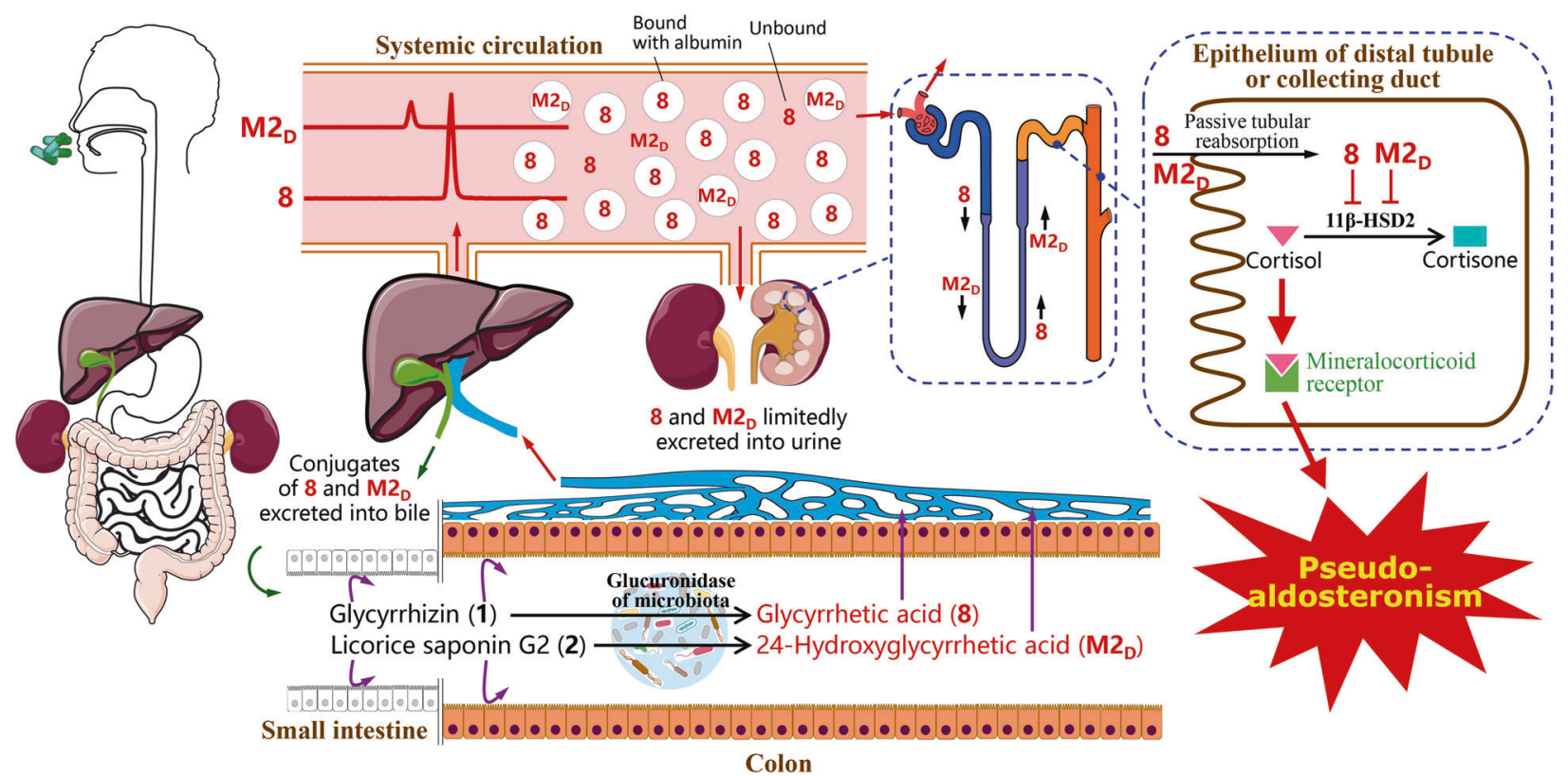

Fig. 8 Schematic overview of pharmacokinetics-based identification of pseudoaldosterogenic compounds glycyrrhetic acid (8) and 24hydroxyglycyrrhetic acid (M2 $\mathbf{D}_{\mathrm{D}}$ ). The Gancao constituents glycyrrhizin (1) and licorice saponin G2 (2) are metabolically activated by glucuronidase of the colonic microbiota to the pseudoaldosterogenic metabolites $\mathbf{8}$ and $\mathbf{M} \mathbf{2}_{\mathbf{D}}$, respectively, which can access (via passive tubular reabsorption) and inhibit renal 11/-HSD2. The finding has implications for precisely defining conditions for safe use of LianhuaQingwen.

circulation after dosing justify such pharmacokinetic research that facilitates uncovering active constituents responsible for the capsule's therapeutic action. Meanwhile, given their significant involvement in acute viral respiratory illnesses, commensal colonic microbiota could be new loci for drug targeting $[27,49,50]$. Unlike systemic exposure, however, colonluminal exposure that allows drug molecules to access colonic microbiota is limitedly investigated in most current pharmacokinetic investigations. Factors governing colon-luminal exposure to Gancao-related compounds after orally dosing LianhuaQingwen included: poor intestinal absorption of the Gancao glycoside constituents [glycyrrhizin (1), licorice saponin G2 (2), and liquiritin/liquiritin apioside (21/22); all BCS Class III compounds] and associated deglycosylation by colonic microbiota to yield their metabolites $\left(\mathbf{8}, \mathbf{M} \mathbf{2}_{\mathrm{D}}\right.$, and $\mathbf{2 7}$, respectively; the first two BCS Class II compounds, the last one BCS Class I compounds). Although all these glycoside constituents and deglycosylated metabolites were detected in human and/or rat fecal samples after dosing LianhuaQingwen, there appeared to be large intercompound differences in colon-luminal residence. Based on their detection in fecal samples, the deglycosylation would shorten such residence time of the glycoside constituents 1, 2, 21, and 22. Regarding the deglycosylated metabolites, colonluminal residence of $\mathbf{8}$ and $\mathbf{M} \mathbf{2}_{\mathbf{D}}$ appeared to be longer than that of 27, mainly due to their differences in BCS Class. Identification of bioavailable Gancao-related compounds with respect to their systemic and colon-luminal exposure, together with on-going identification of such bioavailable compounds originating from other components of LianhuaQingwen, has implications for indepth investigations of the capsule's therapeutic effects, adverse effects, and drug-drug interactions. The bioavailable compounds $\mathbf{8}$ and $\mathbf{2 7}$ have been reported to have antiinflammatory, antiviral, and antimicrobial activities [51, 52], but little is known about bioactivities of $\mathbf{M} \mathbf{2}_{\mathbf{D}}$, particularly those related to COVID-19 treatment.

Gancao-induced pseudoaldosteronism is believed to be related to the Gancao saponin constituent glycyrrhizin and its metabolites [31]. However, which Gancao-related compounds are responsible for the adverse effect remains inconclusive, mainly due to incomplete and inaccurate understanding of systemic exposure to glycyrrhizin and its metabolites and their access to renal $11 \beta$ HSD2. Furthermore, glycyrrhizin is not the only saponin constituent of Gancao [53, 54]. In the current investigation, Gancao saponin constituents and their metabolites were investigated to identify the compounds that could induce pseudoaldosteronism. The identification was based on human systemic exposure to the Gancao compounds after dosing LianhuaQingwen and their access to and inhibition of renal $11 \beta-$ HSD2.

Systemic exposure to Gancao saponins after dosing LianhuaQingwen is governed by the compounds' metabolism, which mainly comprises two steps: (i) colonic deglycosylation of glycyrrhizin (1) and licorice saponin G2 (2), by colonic microbiota, mainly into absorbable glycyrrhetic acid (8) and 24hydroxyglycyrrhetic acid $\left(\mathbf{M 2}_{\mathbf{D}}\right)$, respectively, and (ii) hepatic glucuronidation and sulfation of the absorbed $\mathbf{8}$ and $\mathbf{M} \mathbf{2}_{\mathbf{D}}$ (Fig. 6). Despite being the most abundant Gancao saponin constituents in the capsule, unchanged $\mathbf{1}$ and $\mathbf{2}$ exhibited low systemic exposure, due to their limited intestinal absorption and their rapid first-pass hepatobiliary excretion [44, 55]. Like 8, glycyrrhetic acid-3-Oglucuronide (7; which had been wrongly considered to be the glucuronide of $\mathbf{8}[33,34,36]$ and responsible for Gancao-induced pseudoaldosteronism $[32,33,35])$ was a deglycosylated metabolite of 1, but it is not the saponin's major circulating form, due to minor transformation from 1, limited intestinal absorption (due to poor membrane permeability), and rapid deglycosylation into $\mathbf{8}$. As the respective major circulating forms of $\mathbf{1}$ and $\mathbf{2}$, the deglycosylated metabolites $\mathbf{8}$ and $\mathbf{M} \mathbf{2}_{\mathbf{D}}$, were eliminated from general circulation into bile following hepatic conjugations. Glycyrrhetic acid-30-O-glucuronide $\left(\mathbf{M 8}_{\mathbf{G}}\right)$, rather than $\mathbf{7}$, was the only glucuronide of $\mathbf{8}$ (Fig. 5, Table 1, and Supplementary Table S5). Despite being extensively detected in rat bile, $\mathbf{M} \mathbf{8}_{\mathbf{G}}$ exhibited low level of systemic exposure in humans and rats receiving LianhuaQingwen, mainly due to poor membrane permeability and weak transport by hepatic MRP3 or MRP4 (Supplementary Table S6). Both $\mathbf{8}$ and $\mathbf{M} \mathbf{8}_{\mathbf{G}}$ could be sulfated in the liver into glycyrrhetic acid-3-O-sulfate (M8s; 
previously considered as a biomarker of Gancao-induced pseudoaldosteronism [37, 38]) and glycyrrhetic acid-3-O-sulfate-30-Oglucuronide $\left(\mathbf{M 8}_{\mathbf{G}-\mathbf{s}}\right.$ ), respectively. Like $\mathbf{M} \mathbf{8}_{\mathbf{G}}, \mathbf{M} \mathbf{8}_{\mathbf{s}}$ and $\mathbf{M} \mathbf{8}_{\mathbf{G}-\mathbf{s}}$ were detected in rat bile, but their levels of systemic exposure were low in humans and rats receiving the capsule. Hepatic oxidation of $\mathbf{8}$ yielded $\mathbf{M} \mathbf{2}_{\mathbf{D}}$ and a minor hydroxylated metabolite $\left(\mathbf{M} \mathbf{8}_{\mathbf{O}}\right)$. Both $\mathbf{M 2}_{\mathbf{D}}$ and $\mathbf{M} \mathbf{8}_{\mathbf{O}}$ were glucuronized and sulfated, in the liver, into multiple conjugates $\left(\mathbf{M}_{\mathbf{D}-\mathbf{G}}, \mathbf{M}_{\mathbf{D}-\mathbf{S}}, \mathbf{M} \mathbf{2}_{\mathbf{D}-\mathbf{G}-\mathbf{S}}, \mathbf{M}_{\mathbf{O}-\mathbf{G}}, \mathbf{M}_{\mathbf{O}-\mathbf{S}}\right.$, and M8 o-G-s), all of which could be detected in rat bile samples but not in human and rat plasma samples. Collectively, after dosing LianhuaQingwen, 8 and $\mathbf{M}_{\mathbf{D}}$ were the major circulating Gancao saponin-related compounds; their hepatic conjugated metabolites were mainly excreted into bile, rather than released into the bloodstream.

Renal $11 \beta-H S D 2$ is highly expressed in the epithelia of the distal tubule and collecting duct $[56,57]$. Access of glycyrrhetic acid (8) and 24-hydroxyglycyrrhetic acid (M2 $\mathbf{2}_{\mathbf{D}}$ ) to the renal $11 \beta-\mathrm{HSD} 2$ was evaluated around the renal excretion. Appearance of a drug in the urine is the net result of glomerular filtration, active tubular secretion, and tubular reabsorption. The amount of drug excreted into tubular fluid (urine) via glomerular filtration depends on the systemic exposure level, plasma protein binding, and the GFR. In the proximal tubule, active tubular secretion mediated by basolateral uptake transporters (probably with a joint action of apical efflux transporters) can add the drug into the tubular fluid, while several apical uptake transporters can mediate active tubular reabsorption to reduce the drug in the fluid [58]. In addition, drug having good membrane permeability can undergo passive tubular reabsorption due to concentration gradient for the back-diffusion created by the water reuptake process (particularly in the distal tubule); the degree of the reabsorption is also influenced by the tubular fluid flow and $\mathrm{pH}$ (particularly for weak acids and bases with a $\mathrm{p} K_{\mathrm{a}}$ value of 4.5-8.2). Circulating 8 was excreted into tubular fluid mainly via glomerular filtration, most likely without active tubular secretion and active tubular reabsorption in the proximal tubule. Due to its good membrane permeability, 8 in tubular fluid was reabsorbed into the epithelia by passive diffusion, particularly into the cells of the distal tubule and collecting duct to access $11 \beta-$ HSD2. The tubular reabsorption of 8 was demonstrated by $R_{\mathrm{rc}}$ values of $0.2-0.5$ for human volunteers orally receiving LianhuaQingwen and such values of 0.02 for rats intravenously receiving glycyrrhetic acid. Given its good membrane permeability and limited detection in human urine samples after dosing LianhuaQingwen, circulating $\mathbf{M}_{\mathbf{D}}$ is expected to exhibit a similar scenario regarding access to renal $11 \beta$-HSD2. Urine $\mathrm{pH}$ changes could affect passive tubular reabsorption of $\mathbf{8}$ and $\mathbf{M} \mathbf{2}_{\mathrm{D}},\left(\mathrm{p} K_{\mathrm{a}}, 4.9\right.$; predicted using $\mathrm{ACD} /$ Percepta Platform). Both low systemic exposure and poor membrane permeability limited access of the parent compound glycyrrhizin (1), the conjugated metabolites of $\mathbf{8}$ and M2 $\mathbf{D}$, and $\mathbf{7}$ to renal $11 \beta-\mathrm{HSD} 2$. Regarding Gancao-induced pseudoaldosteronism, previous assessment of $11 \beta-H S D 2$ inhibition by glycyrrhizin and its metabolites by others used the rat enzyme, rather than the human enzyme $[35,39]$. Given possible interspecies differences in $11 \beta$-HSD2 inhibition $[59,60]$, HKM was used to evaluate inhibitory activities of Gancao compounds that were significantly bioavailable at the renal enzyme after dosing LianhuaQingwen. Both 8 and $\mathbf{M} \mathbf{2}_{\mathbf{D}}$ potently inhibited human renal $11 \beta$-HSD2. Notably, significant differences in $I C_{50}$ of test compounds were observed when using HKM from different sources (Celsis product versus Sekisui product); these differences may be due to $11 \beta-H S D 2$ genetic variation. Collectively, the metabolites 8 and $\mathbf{M}_{\mathbf{D}}$ can access and inhibit renal 11 $\beta$-HSD2, with contribution of $\mathbf{8}$ to inducing pseudoaldosteronism greater than that of $\mathbf{M} \mathbf{2}_{\mathbf{D}}$, mainly due to differences in their systemic exposure levels. Although many conjugated metabolites of $\mathbf{8}$ and $\mathbf{M} 2_{\mathbf{D}}$ also inhibit $11 \beta-H S D 2$, they have limited access to the enzyme.
Excessive and prolonged use of Gancao-containing herbal medicines and dietary products, as well as glycyrrhizin formulations, is a main cause of pseudoaldosteronism [31]. Given the widespread utilization of Gancao products, Gancao-induced pseudoaldosteronism risk during administration of LianhuaQingwen can be potentiated when Gancao-containing dietary products and/or other Gancao-containing medicines are concomitantly ingested. Several factors related to the colon, liver, and kidneys can also influence systemic exposure to glycyrrhetic acid (8) and 24hydroxyglycyrrhetic acid (M2 $\mathbf{D}$ ) and/or their access to renal $11 \beta$ HSD2. In the colon, deglycosylations of glycyrrhizin (1) and licorice saponin G2 (2) by microbiota and subsequent absorption of the resulting both BCS Class II compounds govern systemic exposure to these two metabolites after dosing LianhuaQingwen. Colonic microbiota exhibits large interindividual variations in composition and function and can be altered by drugs, herbal compounds, and diet, as well as diseases [61, 62]; this could influence colon-luminal and systemic exposure to $\mathbf{8}$ and $\mathbf{M} \mathbf{2}_{\mathbf{D}}$ [63]. Constipation was found to increase plasma $A U C_{0-48 h}$ of $\mathbf{8}$ and $\mathbf{M}_{\mathbf{D}}$ after dosing LianhuaQingwen, due to, at least in part, the compounds' prolonged colonic absorption. In the liver, the elimination via glucuronidation and sulfation also governs systemic exposure to $\mathbf{8}$ and $\mathbf{M} \mathbf{2}_{\mathbf{D}}$. The hepatic metabolism can be altered by diseases, colonic microbiota, genetic variations of the enzymes, and drug-drug interactions. In the kidneys, glomerular filtration and passive tubular reabsorption, governing access of $\mathbf{8}$ and $\mathbf{M} \mathbf{2}_{\mathrm{D}}$ to renal $11 \beta-H S D 2$, can be affected by changes in overall renal function. Extensive binding to the plasma protein albumin is an important factor limiting the access of 8 and $\mathbf{M} 2_{D}$ to renal $11 \beta-H S D 2$ and in turn contains Gancao-induced pseudoaldosteronism. Accordingly, hepatic synthesis of albumin is important, which could be reduced by hypoalbuminemia (accompanying many diseases, such as chronic hepatitis, cirrhosis, malnutrition, nephrotic syndrome, and sepsis [64]). SARS-CoV-2 infection can cause gastrointestinal symptoms, such as vomiting, diarrhea, or abdominal pain during the early phases of COVID-19 and induce alterations in intestinal microbiome, as well as in metabolic capabilities of the microbial enzymes [28]. Data on clinical characteristics worldwide indicated that around $60 \%$ of COVID-19 positive individuals had one or more preexisting comorbid conditions, such as hypertension, liver diseases, chronic kidney diseases, and metabolic disorders [65]. Given the altered intestinal microbiota and the underlying comorbidity, added precautions should be recommended to prevent Gancao-induced pseudoaldosteronism in such patient populations with comorbidities. To ensure safe use of LianhuaQingwen, (i) increased awareness of Gancaoinduced pseudoaldosteronism and the presence of Gancao in the capsule is required and any pseudoaldosteronism case related to the capsule should be reported; (ii) the capsule should be administered as directed on the leaflets or the prescription and caution should be exercised when the capsule is administered concurrently with other Gancao-containing medicines and dietary products; (iii) caution should be exercised when the capsule is administered in persons with decreased $11 \beta-H S D 2$ activity, hypokalemia, hypertension, impaired liver function, and hypoalbuminemia; and (iv) once pseudoaldosteronism occurs, the capsule and co-administered Gancao-containing products should be withdrawn, and diuretics (such as spironolactone and eplerenone) and drugs (that alkalinize $\mathrm{pH}$ of tubular fluid) may be administered to reduce tubular reabsorption of $\mathbf{8}$ and $\mathbf{M} \mathbf{2}_{\mathrm{D}}$.

In summary, the microbial metabolites glycyrrhetic acid (8), 24hydroxyglycyrrhetic acid (M2 $\mathbf{D}$; a new Gancao metabolite), liquiritigenin (27), and glucuronides of $\mathbf{2 7}\left(\mathbf{M} 27_{\mathbf{G 1}}\right.$ and $\mathbf{M} 27_{\mathbf{G} 2}$ ) are Gancao compounds circulating significantly in humans receiving LianhuaQingwen. The metabolites $\mathbf{8}, \mathbf{M} \mathbf{2}_{\mathrm{D}}$, and $\mathbf{2 7}$ are Gancao compounds exhibiting significant colon-luminal exposure, while their respective unabsorbed parent constituents glycyrrhizin (1), licorice saponin G2 (2), and liquiritin/liquiritin apioside (21/22) also have such exposure but with short residence times in the 
colon. The pharmacokinetics-based identification of these bioavailable Gancao compounds, together with such investigations of other components of LianhuaQingwen, will facilitate uncovering active constituents responsible for the capsule's therapeutic action. In addition, $\mathbf{1}$ and $\mathbf{2}$ are metabolically activated by glucuronidase of colonic microbiota to the pseudoaldosterogenic metabolites 8 and $\mathbf{M 2}_{\mathbf{D}}$ that can access (via passive tubular reabsorption) and potently inhibit renal $11 \beta-H S D 2$ (with large differences in $\mathrm{IC}_{50}$ among human kidney microsomes of different sources) (Fig. 8). Although $\mathbf{8}$ and $\mathbf{M 2}_{\mathbf{D}}$ are not the only Gancaorelated compounds exhibiting inhibitory activities on $11 \beta-H S D 2$, they are the only ones that are bioaccessible for the enzyme. Several factors related to the colon, liver, and kidneys can also influence systemic exposure to $\mathbf{8}$ and $\mathbf{M} \mathbf{2}_{\mathbf{D}}$ and/or the compounds' access to renal $11 \beta$-HSD2. These findings will help in precisely defining conditions for safe use of LianhuaQingwen and also facilitate safety investigations of other Gancao-containing medicines or herbal products.

\section{ACKNOWLEDGEMENTS}

This work was funded by grants from the National Key R\&D Program of China (2018YFC1704500), the National Natural Science Foundation of China (82074273 and 81603380), the Strategic Priority Research Program of the Chinese Academy of Sciences (XDA12050306), and the National Science \& Technology Major Project of China 'Key New Drug Creation and Manufacturing Program' (2017ZX09301012-006). We thank Professor Hai-xue Kuang and Dr. Yan Liu from Heilongjiang University of Chinese Medicine for their kind sharing of their HKM.

\section{AUTHOR CONTRIBUTIONS}

Participated in the research design: $\mathrm{CL}, \mathrm{WWJ}$, and $\mathrm{XFL}$; Conducted the experiments: XFL, WWJ, JLL, FFD, WY, YHS, FOW, XSZ, NNT, XY, FX, HTW, NXZ, and PWL; Performed the data analysis: $C L, W W J, X F L, J L Y, C C, Y F L$, and OEO; Wrote or contributed to the writing of the manuscript: $\mathrm{CL}$, WWJ, OEO, and XFL.

\section{ADDITIONAL INFORMATION}

Supplementary information The online version contains supplementary material available at https://doi.org/10.1038/s41401-021-00651-2.

Competing interests: The authors declare no competing interests.

\section{REFERENCES}

1. Ni LQ, Chen LL, Huang X, Han CP, Xu JR, Zhang H, et al. Combating COVID-19 with integrated traditional Chinese and Western medicine in China. Acta Pharm Sin B. 2020;10:1149-62.

2. Chinese National Health Commission and State Administration of Traditional Chinese Medicine. Diagnosis and treatment protocol for novel coronavirus pneumonia (Trial Version 7), March 3, 2020. (https://www.who.int/docs/default-source/ wpro---documents/countries/china/covid-19-briefing-nhc/1-clinical-protocols-forthe-diagnosis-and-treatment-of-covid-19-v7.pdf?sfvrsn=c6cbfba4_2).

3. Hu K, Guan WJ, Bi Y, Zhang W, Li LJ, Zhang BL, et al. Efficacy and safety of Lianhuaqingwen capsules, a repurposed Chinese herb, in patients with coronavirus disease 2019: a multicenter, prospective, randomized controlled trial. Phytomedicine. 2020;153242.

4. Yao KT, Liu YM, Li X, Huang JH, Cai HB. Retrospective clinical analysis on treatment of novel coronavirus infected pneumonia with traditional Chinese medicine Lianhua Qingwen. Chin J Exp Tradit Med Formula. 2020;26:8-12.

5. Cheng DZ, Wang WJ, Li Y, Wu XD, Zhou B, Song QY. Analysis of curative effect of 51 patients with novel coronavirus pneumonia treated with Chinese medicine Lianhua Qingwen: a multicentre retrospective study. Tianjin J Tradit Chin Med. 2020:37:509-15.

6. Li RF, Hou YL, Huang JC, Pan WQ, Ma QH, Shi YX, et al. Lianhuaqingwen exerts anti-viral and anti-inflammatory activity against novel coronavirus (SARS-CoV-2). Pharmacol Res. 2020;156:104761.

7. Chen XF, Wu YL, Chen C, Gu YQ, Zhu CY, Wang SP, et al. Identifying potential antiCOVID-19 pharmacological components of traditional Chinese medicine Lianhuaqingwen capsule based on human exposure and ACE2 biochromatography screening. Acta Pharm Sin B. 2021;11:222-36.
8. Chinese National Health Commission and Chinese State Administration of Traditional Chinese Medicine. Diagnosis and treatment plan for influenza (2019 version), November 7, 2019. (http://www.nhc.gov.cn/yzygj/s7653p/201911/ a577415af4e5449cb30ecc6511e369c7/files/75a810713dc14dcd9e6db8b654bdef79. pdf).

9. National Health and Family Planning Commission of People's Republic of China. Gulideline on diagnosis and treatment of Middle East respirotory syndrome (2015 version). Chin J Viral Dis. 2015;5:352-54.

10. Lu T, Yang JL, Gao XM, Chen P, Du FF, Sun Y, et al. Plasma and urinary tanshinol from Salvia miltiorrhiza (Danshen) can be used as pharmacokinetic markers for cardiotonic pills, a cardiovascular herbal medicine. Drug Metab Dispos. 2008;36:1578-86.

11. Liu HF, Yang JL, Du FF, Gao XM, Ma XT, Huang YH, et al. Absorption and disposition of ginsenosides after oral administration of Panax notoginseng extract to rats. Drug Metab Dispos. 2009;37:2290-8.

12. Li L, Zhao YS, Du FF, Yang JL, Xu F, Niu W, et al. Intestinal absorption and presystemic elimination of various chemical constituents present in GBE50 extract, a standardized extract of Ginkgo biloba leaves. Curr Drug Metab. 2012;13:494-509.

13. Chen F, Li L, Xu F, Sun Y, Du FF, Ma XT, et al. Systemic and cerebral exposure to and pharmacokinetics of flavonols and terpenelactones after dosing standardized Ginkgo biloba leaf extracts to rats via different routes of administration. Br J Pharmacol. 2013;170:440-57.

14. Hu ZY, Yang JL, Cheng C, Huang YH, Du FF, Wang FQ, et al. Combinatorial metabolism notably affects human systemic exposure to ginsenosides from orally administered extract of Panax notoginseng roots (Sanqi). Drug Metab Dispos. 2013;41:1457-69.

15. Li MJ, Wang FQ, Huang YH, Du FF, Zhong CC, Olaleye OE, et al. Systemic exposure to and disposition of catechols derived from Salvia miltiorrhiza roots (Danshen) after intravenous dosing DanHong injection in human subjects, rats, and dogs. Drug Metab Dispos. 2015;43:679-90.

16. Cheng C, Du F, Yu K, Xu F, Wang FQ, Li L, et al. Pharmacokinetics and disposition of circulating iridoids and organic acids in rats intravenously receiving ReDuNing injection. Drug Metab Dispos. 2016;44:1853-8.

17. Cheng C, Lin JZ, Li L, Yang JL, Jia WW, Huang YH, et al. Pharmacokinetics and disposition of monoterpene glycosides derived from Paeonia lactiflora roots (Chishao) after intravenous dosing of antiseptic XueBiJing injection in human subjects and rats. Acta Pharmacol Sin. 2016;37:530-44.

18. Li XX, Cheng C, Wang FQ, Huang YH, Jia WW, Olaleye OE, et al. Pharmacokinetics of catechols in human subjects intravenously receiving XueBiJing injection, an emerging antiseptic herbal medicine. Drug Metab Pharmacokinet. 2016; 31:95-8.

19. Zhang NT, Cheng C, Olaleye OE, Sun Y, Li L, Huang YH, et al. Pharmacokineticsbased identification of potential therapeutic phthalides from XueBiJing, a Chinese herbal injection used in sepsis management. Drug Metab Dispos. 2018;46:823-34.

20. Zhang HY, Niu W, Olaleye OE, Du FF, Wang FQ, Huang YH, et al. Comparison of intramuscular and intravenous pharmacokinetics of ginsenosides in humans after dosing XueShuanTong, a lyophilized extract of Panax notoginseng roots. J Ethnopharmacol. 2020;253:112658.

21. Liu XW, Yang JL, Niu W, Jia WW, Olaleye OE, Wen Q, et al. Human pharmacokinetics of ginkgo terpene lactones and impact of carboxylation in blood on their plateletactivating factor antagonistic activity. Acta Pharmacol Sin. 2018;39:1935-46.

22. Xie GX, Wang SL, Zhang H, Zhao AH, Liu JJ, Ma YM, et al. Poly-pharmacokinetic study of a multicomponent herbal medicine in healthy Chinese volunteers. Clin Pharmacol Ther. 2018;103:692-702.

23. Olaleye OE, Niu W, Du FF, Wang FQ, Xu F, Pintusophon S, et al. Multiple circulating saponins from intravenous ShenMai inhibit OATP1Bs in vitro: potential joint precipitants of drug interactions. Acta Pharmacol Sin. 2019;40:833-49.

24. Pintusophon $S$, Niu W, Duan $X N$, Olaleye $O E$, Huang $Y H$, Wang $F Q$, et al. Intravenous formulation of Panax notoginseng root extract: human pharmacokinetics of ginsenosides and potential for perpetrating drug interactions. Acta Pharmacol Sin. 2019;40:1351-63.

25. Li J, Olaleye OE, Yu X, Jia WW, Yang JL, Lu C, et al. High degree of pharmacokinetic compatibility exists between the five-herb medicine XueBiJing and antibiotics comedicated in sepsis care. Acta Pharm Sin B. 2019;9:1035-49.

26. Lynch SV, Pedersen O. The human intestinal microbiome in health and disease. $\mathrm{N}$ Engl J Med. 2016;375:2369-79.

27. Hanada S, Pirzadeh M, Carver KY, Deng JC. Respiratory viral infection-induced microbiome alterations and secondary bacterial pneumonia. Front Immunol. 2018;9:2640.

28. Villapol S. Gastrointestinal symptoms associated with COVID-19: impact on the gut microbiome. Transl Res. 2020;226:57-69.

29. Gao QY, Chen YX, Fang JY. 2019 Novel coronavirus infection and gastrointestinal tract. J Dig Dis. 2020;21:125-6. 
30. Cinatl J, Morgenstern B, Bauer G, Chandra P, Rabenau H, Doerr HW. Glycyrrhizin, an active component of liquorice roots, and replication of SARS-associated coronavirus. Lancet. 2003;361:2045-6.

31. Omar HR, Komarova I, El-Ghonemi M, Fathy A, Rashad R, Abdelmalak HD, et al. Licorice abuse: time to send a warning message. Ther Adv Endocrinol Metab. 2012;3:125-38.

32. Kato H, Kanaoka M, Yano S, Kobayashi M. 3-Monoglucuronyl-glycyrrhetinic acid is a major metabolite that causes licorice-induced pseudoaldosteronism. J Clin Endocrinol Metab. 1995;80:1929-33.

33. Ohtake N, Kido A, Kubota K, Tsuchiya N, Morita T, Kase $Y$, et al. A possible involvement of 3-monoglucuronyl-glycyrrhetinic acid, a metabolite of glycyrrhizin (GL), in GL-induced pseudoaldosteronism. Life Sci. 2007;80: 1545-52.

34. Makino T, Ohtake N, Watanabe A, Tsuchiya N, Imamura S, lizuka S, et al. Downregulation of a hepatic transporter multidrug resistance-associated protein 2 is involved in alteration of pharmacokinetics of glycyrrhizin and its metabolites in a rat model of chronic liver injury. Drug Metab Dispos. 2008;36:1438-43.

35. Makino T, Okajima K, Uebayashi R, Ohtake N, Inoue K, Mizukami H. 3Monoglucuronyl-glycyrrhretinic acid is a substrate of organic anion transporters expressed in tubular epithelial cells and plays important roles in licoriceinduced pseudoaldosteronism by inhibiting $11 \beta$-hydroxysteroid dehydrogenase 2. J Pharmacol Exp Ther. 2012;342:297-304.

36. Makino T. 3-Monoglucuronyl glycyrrhretinic acid is a possible marker compound related to licorice-induced pseudoaldosteronism. Biol Pharm Bull. 2014;37: 898-902.

37. Ishiuchi K, Morinaga O, Ohkita T, Tian C-T, Hirasawa A, Mitamur M, et al. 18ßGlycyrrhetyl-3-O-sulfate would be a causative agent of licorice-induced pseudoaldosteronism. Sci Rep. 2019;9:1587.

38. Takahashi K, Yoshino T, Maki Y, Ishiuchi K, Namiki T, Ogawa-Ochiai K, et al. Identification of glycyrrhizin metabolites in humans and of a potential biomarker of liquorice-induced pseudoaldosteronism: a multi-centre cross-sectional study. Arch Toxicol. 2019;93:3111-9.

39. Morinaga $\mathrm{O}$, Ishiuchi $\mathrm{K}$, Ohkita $\mathrm{T}$, Tian $\mathrm{CT}$, Hirasawa $\mathrm{A}$, Mitamur $\mathrm{M}$, et al. Isolation of a novel glycyrrhizin metabolite as a causal candidate compound for pseudoaldosteronism. Sci Rep. 2018;8:15568.

40. McCabe C, Sane RS, Keith-Luzzi M, Xu J, King I, Whitcher-Johnstone A, et al. Defining the role of gut bacteria in the metabolism of deleobuvir: in vitro and in vivo studies. Drug Metab Dispos. 2015;43:1612-8.

41. Yang J-L, He M-M, Niu W, Wrighton SA, Li L, Liu Y, et al. Metabolic capabilities of cytochrome P450 enzymes in Chinese liver microsomes compared with those in Caucasian liver microsomes. Br J Clin Pharmacol. 2012;73:268-84.

42. Tian DD, Jia WW, Liu XW, Wang DD, Liu JH, Dong JJ, et al. Methylation and its role in the disposition of tanshinol, a cardiovascular carboxylic catechol from Salvia miltiorrhiza roots (Danshen). Acta Pharmacol Sin. 2015;36:627-43.

43. Jia WW, Du FF, Liu XW, Jiang RR, Xu F, Yang JL, et al. Renal tubular secretion of tanshinol: molecular mechanisms, impact on its systemic exposure, and propensity for dose-related nephrotoxicity and for renal herb-drug interactions. Drug Metab Dispos. 2015;43:669-78.

44. Dong JJ, Olaleye OE, Jiang RR, Li J, Lu C, Du FF, et al. Glycyrrhizin has a high likelihood to be a victim of drug-drug interactions mediated by hepatic organic aniontransporting polypeptide 1B1/1B3. Br J Pharmacol. 2018;175:3486-503.

45. Guo B, Li C, Wang G-J, Chen L-S. Rapid and direct measurement of free concentrations of highly protein-bound fluoxetine and its metabolite norfluoxetine in plasma. Rapid Commun Mass Spectrom. 2006;20:39-47.
46. Reagan-Shaw S, Nihal M, Ahmad N. Dose translation from animal to human studies revisited. FASEB J. 2008;22:659-61.

47. Brown RW, Chapman KE, Kotelevtsev Y, Yau JL, Lindsay RS, Brett L, et al. Cloning and production of antisera to human placental 11ß-hydroxysteroid dehydrogenase type 2. Biochem J. 1996;313:1007-17.

48. Davies B, Morris T. Physiological parameters in laboratory animals and humans. Pharmacol Res. 1993;10:1093-5.

49. Jia W, Li H, Zhao L-P, Nicholson KJ. Gut microbiota: a potential new territory for drug targeting. Nat Rev Drug Discov. 2008;7:123-9.

50. Cani PD, Delzenne NM. The gut microbiome as therapeutic target. Pharmacol Ther. 2011;130:202-12.

51. Kowalska A, Kalinowska-Lis U. 18ß-Glycyrrhetinic acid: its core biological properties and dermatological applications. Int J Cosmet Sci. 2019;41:325-31.

52. Ramalingam M, Kim H, Lee Y, Lee YI. Phytochemical and pharmacological role of Liquiritigenin and isoliquiritigenin from radix glycyrrhizae in human health and disease models. Front Aging Neurosci. 2018;10:348.

53. Zhang $\mathrm{Q}, \mathrm{Ye} \mathrm{M}$. Chemical analysis of the Chinese herbal medicine Gan-Cao (licorice). J Chromatogr A. 2009;1216:1954-69.

54. Qiao X, Song W, Ji S, Wang Q, Guo D-A, Ye M. Separation and characterization of phenolic compounds and triterpenoid saponins in licorice (Glycyrrhiza uralensis) using mobile phase-dependent reversed-phase $\times$ reversed-phase comprehensive two-dimensional liquid chromatography coupled with mass spectrometry. J Chromatogr A. 2015;1402:36-45.

55. Yu K, Chen F, Li C. Absorption, disposition, and pharmacokinetics of saponins from Chinese medicinal herbs: what do we know and what do we need to know more?. Curr Drug Metab. 2012;13:577-98.

56. Chapman K, Holmes M, Seckl J. 11ß-hydroxysteroid dehydrogenases: intracellular gate-keepers of tissue glucocorticoid action. Physiol Rev. 2013;93: 1139-206.

57. Hunter RW, Bailey MA. Glucocorticoids and $11 \beta$-hydroxysteroid dehydrogenases: mechanisms for hypertension. Curr Opin Pharmacol. 2015;21:105-14.

58. Morrissey KM, Stocker SL, Wittwer MB, Xu L, Giacomini KM. Renal transporters in drug development. Annu Rev Pharmacol Toxicol. 2013;53:503-29.

59. Beck KR, Bächler M, Vuorinen A, Wagner S, Akram M, Griesser U, et al. Inhibition of $11 \beta$-hydroxysteroid dehydrogenase 2 by the fungicides itraconazole and posaconazole. Biochem Pharmacol. 2017;130:93-103.

60. Kratschmar DV, Vuorinen A, Cunha TD, Wolber G, Classen-Houben D, Doblhoff O, et al. Characterization of activity and binding mode of glycyrrhetinic acid derivatives inhibiting 11ß-hydroxysteroid dehydrogenase type 2. J Steroid Biochem Mol Biol. 2011;125:129-42.

61. Maier L, Pruteanu M, Kuhn M, Zeller G, Telzerow A, Anderson EE, et al. Extensive impact of non-antibiotic drugs on human gut bacteria. Nature. 2018;555: 623-8.

62. Gentile $\mathrm{CL}$, Weir TL. The gut microbiota at the intersection of diet and human health. Science. 2018;362:776-80.

63. Liu M, Yuan J, Hu WJ, Ke CQ, Zhang YF, Yang YE, et al. Pretreatment with broadspectrum antibiotics alters the pharmacokinetics of major constituents of Shaoyao-Gancao decoction in rats after oral administration. Acta Pharmacol Sin. 2019;40:288-96.

64. Gatta A, Verardo A, Bolognesi M. Hypoalbuminemia. Intern Emerg Med. 2012;7: S193-9.

65. Bajgain KT, Badal S, Bajgain BB, Santana MJ. Prevalence of comorbidities among individuals with COVID-19: A rapid review of current literature. Am J Infect Control. 2021;49:238-46. 